\title{
Nonreflecting Boundary Conditions for Elastodynamic Scattering
}

\section{Report}

Author(s):

Grote, Marcus J.

Publication date:

1999-10

Permanent link:

https://doi.org/10.3929/ethz-a-004284981

Rights / license:

In Copyright - Non-Commercial Use Permitted

Originally published in:

SAM Research Report 1999-19 


\title{
Nonreflecting Boundary Conditions For Elastodynamic Scattering
}

\author{
M.J. Grote
}

Research Report No. 99-19

October 1999

Seminar für Angewandte Mathematik

Eidgenössische Technische Hochschule

CH-8092 Zürich

Switzerland 


\title{
Nonreflecting Boundary Conditions For Elastodynamic Scattering
}

\author{
M.J. Grote \\ Seminar für Angewandte Mathematik \\ Eidgenössische Technische Hochschule \\ CH-8092 Zürich \\ Switzerland
}

Research Report No. 99-19 October 1999

\begin{abstract}
An exact nonreflecting boundary condition was derived previously for time-dependent elastic waves in three space dimensions [1]. It is local in time, nonlocal on the artificial boundary, and involves only first derivatives of the displacement. Here it is shown how to combine that boundary condition with finite difference and finite element methods. Stability issues are discussed. Numerical examples demonstrate the high improvement in accuracy over standard methods.
\end{abstract}

Keywords: elastic waves, wave propagation, nonreflecting boundary conditions, absorbing boundary conditions, elasticity, scattering theory

Subject Classification (1991): 35L15, 65M99, 73D15, 73D30

submitted to J. Comp. Phys. 


\section{Introduction}

We wish to calculate numerically the time dependent wave field $\boldsymbol{u}(\boldsymbol{x}, t)$ scattered from a bounded scattering region in an unbounded three-dimensional elastic medium. In this region, there may be one or more scatterers and the equation for the displacement $\boldsymbol{u}$ may have variable coefficients and nonlinear terms. As usual, we surround the scattering region by an artificial boundary $\mathcal{B}$ and confine the computation to the region $\Omega$ bounded by $\mathcal{B}$. Then, to complete the formulation of the problem in $\Omega$ we require that $\boldsymbol{u}$ satisfy a boundary condition on $\mathcal{B}$. The boundary conditions commonly imposed produce spurious reflection from $\mathcal{B}$. To avoid this spurious reflection we have devised an exact nonreflecting boundary condition [1]. It is the extension to the elastic wave equation of the exact nonreflecting boundary condition which we derived for the scalar wave equation [2, 3] and for Maxwell's equations [4]. In doing so, we chose $\mathcal{B}$ to be a sphere of radius $R$, and we assumed that the elastic medium is homogeneous and isotropic outside $\mathcal{B}$. The boundary condition is local in time and nonlocal on $\mathcal{B}$, and it involves only first derivatives of $\boldsymbol{u}$ on $\mathcal{B}$.

Usually various approximate boundary conditions are used, which are local differential operators on $\mathcal{B}$ - see for instance Givoli [5] or the recent review article by Tsynkov [6]. Well-known examples are the 'viscous' boundary conditions by Lysmer and Kuhlemeyer [7], and the paraxial boundary conditions by Clayton and Engquist [8] and Engquist and Majda [9, 10]. Higdon [11, 12] constructed absorbing boundary conditions, which perfectly annihilate impinging waves at selected angles of incidence, but not at others, by combining first-order differential operators in time and the normal space variable. Earlier Lindman [13] devised a non-local absorbing boundary condition for the scalar wave equation. It requires solving the inhomogeneous wave equation on the artificial boundary a number of times. Randall $[14,15]$ extended it to the elastic wave equation by applying the absorbing boundary condition of Lindman to a decomposition of the displacement into potentials which satisfy acoustic wave equations; this procedure requires at each time step a Fourier transform in the tangential space variables.

A different approach to eliminating reflection has been to append an artificial transition layer outside $\mathcal{B}$, which is supposed to absorb outgoing waves. Two popular methods for doing this, the mapping technique [16] and the perfectly matched layer method [17], were adapted recently to the absorbtion of elastic waves, and they yielded comparable results [18].

Neither the local boundary conditions nor the use of absorbing layers leads to complete absorption of waves at all angles of incidence. Although most approximate boundary conditions perform well at nearly normal incidence, their performance degrades rapidly as grazing incidence is approached. In complex situations the scattered waves arrive at the artificial boundary from all interior angles and at all frequencies, so these methods then yield some spurious reflection. Moreover, errors due to spurious reflection accumulate with time and prevent accurate long-time integration. Thus it is often necessary to move $\mathcal{B}$ far from the region of interest, or to use a thick absorbing layer, to reduce the amount of reflection below 
a few percent and to achieve high accuracy. Another difficulty is that approximate boundary conditions can result in ill-posed formulations - see Howell and Trefethen [19].

Some of these difficulties are avoided by exact nonreflecting boundary conditions. In the frequency domain, Givoli and Keller [20] derived a Dirichlet-to-Neumann map for twodimensional elastodynamics; it was further developed by Harari and Shohet [21]. In the time domain, an exact nonreflecting boundary condition for the wave equation was proposed by Ting and Miksis [22] and later implemented by Givoli and Cohen [23]. It is based on a Kirchhoff integral representation of the solution on $\mathcal{B}$ and requires storing the solution at a surface inside $\mathcal{B}$ for the length of time it takes a wave to propagate across $\Omega$. To update the solution value at any point on the two-dimensional artificial boundary $\mathcal{B}$ requires computing a two-dimensional integral in space and time. Therefore using this boundary condition may be more expensive than using the numerical scheme itself inside $\Omega$.

It is to avoid the various difficulties mentioned above that we developed the exact nonreflecting boundary condition for the special case when $\mathcal{B}$ is a sphere [1]. Now we shall show how to combine this boundary condition with the finite difference method, or with the finite element method, to obtain a computational problem in $\Omega$. We shall also examine the stability of the ordinary differential equations which occur in the boundary condition. Finally, we shall solve numerically two standard test problems by using an explicit finite difference method and the nonreflecting boundary condition. We shall also solve the same problems with the local boundary condition of Lysmer and Kuhlemeyer [7]. Comparison of these solutions with the "exact" solution, obtained in a very large domain so that spurious reflections are postponed, shows that our boundary condition is much more accurate. We also show that it remains accurate when the artificial boundary is moved closer to the scatterer, so that the computational domain is reduced.

\section{Displacement Formulation}

We consider time-dependent scattering from a bounded scattering region in three-dimensional space. We surround this region by a sphere $\mathcal{B}$ of radius $R$. Outside $\mathcal{B}$, we assume that the elastic medium is homogeneous and isotropic, with constant density $\rho_{0}$ and Lamé constants $\lambda$ and $\mu$. In addition, we assume that at $t=0$ the scattered field is confined to the computational domain $\Omega$, which is the interior of $\mathcal{B}$. Outside $\mathcal{B}$ the scattered displacement field $\boldsymbol{u}(\boldsymbol{x}, t)$ satisfies the elastic wave equation $([24])$,

$$
\frac{\partial^{2} \boldsymbol{u}}{\partial t^{2}}-c_{p}^{2} \nabla \nabla \cdot \boldsymbol{u}+c_{s}^{2} \nabla \times \nabla \times \boldsymbol{u}=0
$$

with initial conditions

$$
\boldsymbol{u}=0, \quad \frac{\partial \boldsymbol{u}}{\partial t}=0, \quad t=0
$$


Here $c_{p}$ and $c_{s}$ are the propagation speeds of compressional waves and shear waves, respectively,

$$
c_{p}^{2}=\frac{\lambda+2 \mu}{\rho_{0}}, \quad c_{s}^{2}=\frac{\mu}{\rho_{0}} .
$$

In $\Omega$, we consider the following initial-boundary value problem problem:

$$
\begin{array}{ll}
\rho \frac{\partial^{2} \boldsymbol{u}}{\partial t^{2}}-2 \mu \Delta \boldsymbol{u}-\lambda \nabla \nabla \cdot \boldsymbol{u}-\mu \nabla \times \nabla \times \boldsymbol{u}=f, & \text { in } \Omega \times(0, T), \\
\boldsymbol{u}(\boldsymbol{x}, 0)=\boldsymbol{u}_{0}(\boldsymbol{x}), \quad \frac{\partial \boldsymbol{u}(\boldsymbol{x}, 0)}{\partial t}=\boldsymbol{v}_{0}, & \boldsymbol{x} \in \Omega .
\end{array}
$$

When $f=0$ and $\mu, \lambda$, and $\rho$ are constant, equation (2.4) is equivalent to (2.1). On $\mathcal{B}$ we impose the exact nonreflecting boundary condition derived by Grote and Keller [1]:

$$
\begin{array}{lc}
\frac{\partial \boldsymbol{u}}{\partial r}+\frac{\boldsymbol{u}}{R}+\frac{1}{c_{s}} \frac{\partial \boldsymbol{u}^{\mathrm{tan}}}{\partial t}+\frac{\hat{\boldsymbol{r}}}{c_{p}} \frac{\partial u^{r}}{\partial t}+\frac{c_{p}-c_{s}}{c_{s}} \hat{\boldsymbol{r}} \times \nabla \times\left(\hat{\boldsymbol{r}} u^{r}\right)+\frac{c_{p}-c_{s}}{c_{p}} \hat{\boldsymbol{r}} \nabla \cdot \boldsymbol{u}^{\text {tan }} \\
=-\frac{1}{R^{2}} \sum_{n \geq 1} \sum_{|m| \leq n}\left\{\boldsymbol{d}_{n} \cdot \boldsymbol{\psi}_{n m} \boldsymbol{V}_{n m}+\boldsymbol{a}_{n} \cdot \boldsymbol{\Psi}_{n m} \boldsymbol{U}_{n m}\right\} & \\
+\frac{1}{R^{2}} \sum_{n \geq 0} \sum_{|m| \leq n} \boldsymbol{b}_{n} \cdot \boldsymbol{\Psi}_{n m} Y_{n m} \hat{\boldsymbol{r}}, & \text { on } \mathcal{B} \times(0, T), \\
\frac{d}{d t} \boldsymbol{\psi}_{n m}=\frac{1}{R} \boldsymbol{A}_{n} \boldsymbol{\psi}_{n m}+\left(\left.\boldsymbol{u}^{\mathrm{tan}}\right|_{r=R}, \boldsymbol{V}_{n m}\right) \boldsymbol{e}_{n}, & \boldsymbol{\psi}_{n m}(0)=0, \\
\frac{d}{d t} \boldsymbol{\Psi}_{n m}=\frac{1}{R} \boldsymbol{B}_{n} \boldsymbol{\Psi}_{n m}+\left[\begin{array}{c}
c_{s}\left(\left.\boldsymbol{u}^{\mathrm{tan}}\right|_{r=R}, \boldsymbol{U}_{n m}\right) \boldsymbol{e}_{n+1} \\
-c_{p}\left(\left.u^{r}\right|_{r=R}, Y_{n m}\right) \boldsymbol{e}_{n+1}
\end{array}\right], & \boldsymbol{\Psi}_{n m}(0)=0 .
\end{array}
$$

Here we have introduced the polar coordinates $r, \vartheta, \phi$ and the unit vectors $\hat{\boldsymbol{r}}, \hat{\boldsymbol{\vartheta}}$, $\hat{\boldsymbol{\phi}}$, while $u^{r}$ and $\boldsymbol{u}^{t a n}$ denote the normal and tangential components of $\boldsymbol{u}=\left(u^{r}, u^{\vartheta}, u^{\phi}\right)$,

$$
\boldsymbol{u}^{\tan }=\left(\begin{array}{c}
0 \\
u^{\vartheta} \\
u^{\phi}
\end{array}\right), \quad \hat{\boldsymbol{r}} u^{r}=\left(\begin{array}{c}
u^{r} \\
0 \\
0
\end{array}\right)
$$

Furthermore, $Y_{n m}$ denotes the $n m$-th spherical harmonic normalized over the unit sphere,

$$
Y_{n m}(\vartheta, \phi)=\sqrt{\frac{(2 n+1)(n-|m|) !}{4 \pi(n+|m|) !}} P_{n}^{|m|}(\cos \vartheta) e^{i m \phi}, \quad n \geq 0,|m| \leq n .
$$

If the problem considered is real, it is advantageous to use the real spherical harmonics, given by the real and imaginary parts of (2.10). Then everything remains the same except for the normalization constant in (2.10), which must be multiplied by $\sqrt{2}$ for $m \neq 0$. The vector spherical harmonics $\boldsymbol{U}_{n m}$ and $\boldsymbol{V}_{n m}$ are defined by

$$
\begin{aligned}
\boldsymbol{U}_{n m}(\vartheta, \phi) & =\frac{r \nabla Y_{n m}}{\sqrt{n(n+1)}}=\frac{1}{\sqrt{n(n+1)}}\left[\frac{\partial Y_{n m}}{\partial \vartheta} \hat{\boldsymbol{\vartheta}}+\frac{1}{\sin \vartheta} \frac{\partial Y_{n m}}{\partial \phi} \hat{\boldsymbol{\phi}}\right], n \geq 1 \\
\boldsymbol{V}_{n m}(\vartheta, \phi) & =\hat{\boldsymbol{r}} \times \boldsymbol{U}_{n m}=\frac{1}{\sqrt{n(n+1)}}\left[\frac{-1}{\sin \vartheta} \frac{\partial Y_{n m}}{\partial \phi} \hat{\boldsymbol{\vartheta}}+\frac{\partial Y_{n m}}{\partial \vartheta} \hat{\boldsymbol{\phi}}\right], \quad n \geq 1 .
\end{aligned}
$$


They form an orthonormal basis for the space of tangential $L_{2}$ fields on the unit sphere with respect to the $L_{2}$ inner product ([25]). In (2.4) we assume that $\lambda$ and $\mu$ are positive constants and require that $\rho=\rho(\boldsymbol{x})>0$. The source term $f(\boldsymbol{x}, t, \boldsymbol{u}, \nabla \boldsymbol{u})$ may be nonlinear.

Equation (2.6) is the exact nonreflecting boundary condition which was derived in [1]. It involves the vector functions $\boldsymbol{\psi}_{n m}(t)$ and $\boldsymbol{\Psi}_{n m}(t)$, which are solutions of the linear first-order ordinary differential equations (2.7) and (2.8). In (2.7) and (2.8) $\boldsymbol{e}_{n}$ is the $n$-component unit vector

$$
\boldsymbol{e}_{n}=[1,0, \ldots, 0]^{\top} .
$$

To simplify the definitions of the remaining quantities we first let

$$
\gamma_{n}=n(n+1), \quad n \geq 0 .
$$

Then $\boldsymbol{d}_{n}=\left\{d_{n}(j)\right\}$ denotes the constant $n$-component vector

$$
d_{n}(j)=\frac{j \gamma_{n}}{2}, \quad j=1, \ldots, n,
$$

The constant 2(n+1)-component vectors $\boldsymbol{a}_{n}$ and $\boldsymbol{b}_{n}$ are given by

$$
\boldsymbol{a}_{n}=\left[\begin{array}{l}
\boldsymbol{a}_{n}^{1} \\
\boldsymbol{a}_{n}^{2}
\end{array}\right], \quad \boldsymbol{b}_{n}=\left[\begin{array}{l}
\boldsymbol{b}_{n}^{1} \\
\boldsymbol{b}_{n}^{2}
\end{array}\right]
$$

where the $(n+1)$-component vectors $\boldsymbol{a}_{n}^{1}=\left\{a_{n}^{1}(j)\right\}$ and $\boldsymbol{a}_{n}^{2}=\left\{a_{n}^{2}(j)\right\}, j=1, \ldots, n+1$, are

$$
a_{n}^{1}(j)=\left[\frac{1}{2}-\frac{1-c_{p} / c_{s}}{1+\gamma_{j-1} / \gamma_{n}}\right] j \gamma_{n}, \quad a_{n}^{2}(j)=\frac{\sqrt{\gamma_{n}} j^{2} c_{p} / c_{s}}{1+\gamma_{j-1} / \gamma_{n}} .
$$

and the $(n+1)$-component vectors $\boldsymbol{b}_{n}^{1}=\left\{b_{n}^{1}(j)\right\}$ and $\boldsymbol{b}_{n}^{2}=\left\{b_{n}^{2}(j)\right\}, j=1, \ldots, n+1$, are

$$
b_{n}^{1}(j)=\frac{j\left(1+(j-1) c_{s} / c_{p}\right) \sqrt{\gamma_{n}}}{1+\gamma_{j-1} / \gamma_{n}}, \quad b_{n}^{2}(j)=\left[\frac{1}{2}+\frac{j-\left(1-c_{s} / c_{p}\right) \gamma_{n}}{\gamma_{n}+\gamma_{j-1}}\right] j \gamma_{n},
$$

In (2.7) the constant $n \times n$ matrix $\boldsymbol{A}_{n}=\left\{A_{n}(i, j)\right\}$ is

$$
A_{n}(i, j)= \begin{cases}-c_{s} \gamma_{n} / 2 & \text { if } i=1 \\ c_{s}\left(\gamma_{n}-\gamma_{j}\right) /(2(j+1)) & \text { if } i=j+1 \\ 0 & \text { otherwise }\end{cases}
$$

Finally, in $(2.8)$ the constant $2(n+1) \times 2(n+1)$ matrix $\boldsymbol{B}_{n}$ consists of the following block partition,

$$
\boldsymbol{B}_{n}=\left[\begin{array}{c|c}
c_{s} \boldsymbol{S}_{n} & -c_{s} \boldsymbol{z}_{n}^{\top} \\
& 0 \\
\hline-c_{p} \boldsymbol{z}_{n}^{\top} & \multirow{2}{*}{c_{p} \boldsymbol{T}_{n}}
\end{array}\right],
$$


where the $(n+1)$-component vector $\boldsymbol{z}_{n}=\left\{z_{n}(j)\right\}$ is defined by

$$
z_{n}(j)=\frac{j \sqrt{\gamma_{n}}}{1+\gamma_{j-1} / \gamma_{n}}, \quad j=1, \ldots, n+1 .
$$

The $(n+1) \times(n+1)$ matrix $\boldsymbol{S}_{n}=\left\{S_{n}(i, j)\right\}$ is

$$
S_{n}(i, j)= \begin{cases}-\gamma_{n} / 2 & \text { if } i=1 \\ \left(\gamma_{n}+\gamma_{j}\right)\left(\gamma_{n}-\gamma_{j-1}\right) /\left[2(j+1)\left(\gamma_{n}+\gamma_{j-1}\right)\right] & \text { if } i=j+1 \\ 0 & \text { otherwise }\end{cases}
$$

and the $(n+1) \times(n+1)$ matrix $\boldsymbol{T}_{n}=\left\{T_{n}(i, j)\right\}$ is

$$
T_{n}(i, j)= \begin{cases}-\left[1+2 j /\left(\gamma_{n}+\gamma_{j-1}\right)\right] \gamma_{n} / 2 & \text { if } i=1 \\ \left(\gamma_{n}+\gamma_{j}\right)\left(\gamma_{n}-\gamma_{j-1}\right) /\left[2(j+1)\left(\gamma_{n}+\gamma_{j-1}\right)\right] & \text { if } i=j+1 \\ 0 & \text { otherwise }\end{cases}
$$

The definitions of $\boldsymbol{A}_{n}, \boldsymbol{S}_{n}, \boldsymbol{T}_{n}, \boldsymbol{d}_{n}, \boldsymbol{e}_{n}$, and $\boldsymbol{z}_{n}$ coincide with those used in [1], except for an improved scaling in inverse powers of $R$, suggested by Thompson and Huan [26] for the scalar wave equation. This new scaling has no effect if $R=1$. The constants $\boldsymbol{a}_{n}^{1}, \boldsymbol{a}_{n}^{2}, \boldsymbol{b}_{n}^{1}$, and $\boldsymbol{b}_{n}^{2}$ were previously denoted in [1] by $\boldsymbol{q}_{n}, \boldsymbol{p}_{n}, \boldsymbol{b}_{n}$, and $\boldsymbol{a}_{n}$, respectively.

The somewhat special case $n=0$ corresponds to the 0 -th Fourier component of the displacement field, which consists of a spherically symmetric compression wave, $\boldsymbol{u}=\hat{\boldsymbol{r}} u^{r}(r, t)$. Thus the first component of $\boldsymbol{\Psi}_{0}$ vanishes and the only nonzero constants needed in (2.6) and (2.8) for $n=0$ are

$$
\boldsymbol{b}_{0}=\left[\begin{array}{l}
0 \\
1
\end{array}\right], \quad \boldsymbol{B}_{0}=\left[\begin{array}{cc}
0 & 0 \\
0 & -c_{p}
\end{array}\right] \text {. }
$$

Suppose that the initial value problem (2.4)-(2.5), with $\Omega$ replaced by $\mathbb{R}^{3}$, has a unique smooth solution. Then so does the initial-boundary value problem (2.4)-(2.8) and the two solutions coincide in $\Omega([1])$. The Cauchy problem (2.4)-(2.5), with $\Omega$ replaced by $\mathbb{R}^{3}$ and $f \equiv 0$, is well-posed with respect to the initial data $\boldsymbol{u}_{0}$ and $\boldsymbol{v}_{0}$. Since its solution coincides with that of the initial-boundary value problem (2.4)-(2.8), we immediately conclude that $(2.4)-(2.8)$ is well-posed with respect to $\boldsymbol{u}_{0}$ and $\boldsymbol{v}_{0}$ when $f=0$.

\section{Finite Element Formulation}

We shall now derive the weak formulation of $(2.4)-(2.8)$ in the computational domain $\Omega$. First, we let $\mathcal{V}=\left[H^{1}(\Omega)\right]^{3}$, the Sobolev space of square-integrable vector functions with square-integrable first derivatives, and we denote by $(.,$.$) and (., .)_{\mathcal{B}}$ the $L_{2}$ inner products over $\Omega$ and $\mathcal{B}$, respectively. To derive the weak formulation we multiply (2.4) by a test function $\boldsymbol{w}$ and integrate over $\Omega$. Then we use integration by parts to obtain

$$
\begin{aligned}
\left(\boldsymbol{w}, \rho \partial_{t t} \boldsymbol{u}\right)+ & 2 \mu(\nabla \boldsymbol{w}, \nabla \boldsymbol{u})+\lambda(\nabla \cdot \boldsymbol{w}, \nabla \cdot \boldsymbol{u})+\mu(\nabla \times \boldsymbol{w}, \nabla \times \boldsymbol{u}) \\
& =(\boldsymbol{w}, f)+(\boldsymbol{w}, \boldsymbol{T} \hat{\boldsymbol{r}})_{\mathcal{B}} .
\end{aligned}
$$


Here $\boldsymbol{T} \hat{\boldsymbol{r}}$ is the traction on $\mathcal{B}$,

$$
\boldsymbol{T} \hat{\boldsymbol{r}}=2 \mu \frac{\partial \boldsymbol{u}}{\partial r}+\lambda \hat{\boldsymbol{r}} \nabla \cdot \boldsymbol{u}+\mu \hat{\boldsymbol{r}} \times \nabla \times \boldsymbol{u} .
$$

The traction requires a priori unknown radial derivatives of $\boldsymbol{u}$, which we shall now express in terms of known quantities. First, we write (3.2) componentwise as

$$
\begin{aligned}
(\boldsymbol{T} \hat{\boldsymbol{r}})^{r} & =(2 \mu+\lambda) \partial_{r} u^{r}+\frac{2 \lambda}{R} u^{r}+\lambda \nabla \cdot \boldsymbol{u}^{\tan } \\
(\boldsymbol{T} \hat{\boldsymbol{r}})^{\tan } & =\mu\left(\partial_{r} \boldsymbol{u}^{\tan }-\frac{\boldsymbol{u}^{\tan }}{R}+\nabla^{\tan } u^{r}\right)
\end{aligned}
$$

Similarly, we rewrite the boundary condition (2.6) as

$$
\begin{aligned}
& \partial_{r} u^{r}+\frac{1}{c_{p}} \partial_{t} u^{r}+\frac{u^{r}}{R}+\frac{c_{p}-c_{s}}{c_{p}} \nabla \cdot \boldsymbol{u}^{\tan }=g^{r}, \\
& \partial_{r} \boldsymbol{u}^{\tan }+\frac{1}{c_{s}} \partial_{t} \boldsymbol{u}^{\tan }+\frac{\boldsymbol{u}^{\tan }}{R}+\frac{c_{p}-c_{s}}{c_{s}} \nabla^{\tan } u^{r}=\boldsymbol{g}^{\tan },
\end{aligned}
$$

where $\boldsymbol{g}(\vartheta, \phi, t)$ denotes the right side of (2.6). Next, we use (3.5) in (3.3) and (3.6) in (3.4) to eliminate $\partial_{r} \boldsymbol{u}$. Thus,

$$
\begin{aligned}
\frac{1}{\rho_{0}}(\boldsymbol{T} \hat{\boldsymbol{r}})^{r} & =c_{p}^{2} g^{r}-c_{p} \partial_{t} u^{r}+\frac{2\left(c_{p}^{2}-2 c_{s}^{2}\right)}{R} u^{r}+c_{s}\left(c_{p}-2 c_{s}\right) \nabla \cdot \boldsymbol{u}^{\tan }, \\
\frac{1}{\rho_{0}}(\boldsymbol{T} \hat{\boldsymbol{r}})^{\tan } & =c_{s}^{2} \boldsymbol{g}^{\tan }-c_{s} \partial_{t} \boldsymbol{u}^{\tan }-c_{s}^{2} \frac{\boldsymbol{u}^{\tan }}{R}-c_{s}\left(c_{p}-2 c_{s}\right) \nabla^{\tan } u^{r} .
\end{aligned}
$$

To simplify the notation we define the two symmetric bilinear forms on $\mathcal{V}$ :

$$
\begin{aligned}
\mathcal{A}[\boldsymbol{w}, \boldsymbol{v}] & =2 \mu(\nabla \boldsymbol{w}, \nabla \boldsymbol{v})+\lambda(\nabla \cdot \boldsymbol{w}, \nabla \cdot \boldsymbol{v})+\mu(\nabla \times \boldsymbol{w}, \nabla \times \boldsymbol{v}) \\
& +\frac{\mu}{R}\left(\boldsymbol{w}^{\tan }, \boldsymbol{v}^{\tan }\right)_{\mathcal{B}}-\frac{2 \lambda}{R}\left(w^{r}, v^{r}\right)_{\mathcal{B}}+c_{s}\left(c_{p}-2 c_{s}\right) \rho_{0}\left(\left(\boldsymbol{w}^{\tan }, \nabla^{\tan } v^{r}\right)_{\mathcal{B}}+\left(\nabla^{\tan } w^{r}, \boldsymbol{v}^{\tan }\right)_{\mathcal{B}}\right) \\
\mathcal{C}[\boldsymbol{w}, \boldsymbol{v}] & =c_{p} \rho_{0}\left(w^{r}, v^{r}\right)_{\mathcal{B}}+c_{s} \rho_{0}\left(\boldsymbol{w}^{\tan }, \boldsymbol{v}^{\tan }\right)_{\mathcal{B}} .
\end{aligned}
$$

We remark that the nonreflecting boundary condition affects the bilinear form $\mathcal{A}$ only if the supports of both $\boldsymbol{v}$ and $\boldsymbol{w}$ contain part of the artificial boundary $\mathcal{B}$. Similarly, the bilinear form $\mathcal{C}$ is zero except for functions $\boldsymbol{v}$ and $\boldsymbol{w}$ whose support intersects $\mathcal{B}$. We now replace the traction in (3.1) by using (3.7) and (3.8) and collect terms that involve $\boldsymbol{u}, \partial_{t} \boldsymbol{u}$, or $\partial_{t t} \boldsymbol{u}$. Moreover, by applying integration by parts over $\mathcal{B}$, we replace $-\left(w^{r}, \nabla \cdot \boldsymbol{u}^{\tan }\right)_{\mathcal{B}}$ by $\left(\nabla^{\tan } w^{r}, \boldsymbol{u}^{\tan }\right)_{\mathcal{B}}$; no additional boundary integral appears because $\mathcal{B}$ has no boundary. These calculations lead to the weak form of the problem, which can be stated as follows: 
Find $\boldsymbol{u}(t) \in \mathcal{V}$ such that for all $\boldsymbol{w} \in \mathcal{V}$,

$$
\begin{aligned}
&(\boldsymbol{w}, \rho \ddot{\boldsymbol{u}})+\mathcal{C}[\boldsymbol{w}, \dot{\boldsymbol{u}}]+\mathcal{A}[\boldsymbol{w}, \boldsymbol{u}]=(\boldsymbol{w}, f)+\frac{2 \mu+\lambda}{R^{2}} \sum_{n \geq 0} \sum_{|m| \leq n} \boldsymbol{b}_{n} \cdot \boldsymbol{\Psi}_{n m}\left(w^{r}, Y_{n m}\right)_{\mathcal{B}} \\
&-\frac{\mu}{R^{2}} \sum_{n \geq 1} \sum_{|m| \leq n}\left\{\boldsymbol{d}_{n} \cdot \boldsymbol{\psi}_{n m}\left(\boldsymbol{w}^{\mathrm{tan}}, \boldsymbol{V}_{n m}\right)_{\mathcal{B}}+\boldsymbol{a}_{n} \cdot \boldsymbol{\Psi}_{n m}\left(\boldsymbol{w}^{\mathrm{tan}}, \boldsymbol{U}_{n m}\right)_{\mathcal{B}}\right\} \\
&(\boldsymbol{w}, \boldsymbol{u}(0, \cdot))=\left(\boldsymbol{w}, \boldsymbol{u}_{0}\right) \\
&(\boldsymbol{w}, \dot{\boldsymbol{u}}(0, \cdot))=\left(\boldsymbol{w}, \boldsymbol{v}_{0}\right), \\
& \dot{\boldsymbol{\psi}}_{n m}=\frac{1}{R} \boldsymbol{A}_{n} \boldsymbol{\psi}_{n m}+\left(\left.\boldsymbol{u}^{\mathrm{tan}}\right|_{r=R}, \boldsymbol{V}_{n m}\right) \boldsymbol{e}_{n}, \\
& \dot{\boldsymbol{\Psi}}_{n m}=\frac{1}{R} \boldsymbol{B}_{n} \boldsymbol{\Psi}_{n m}+\left[\begin{array}{c}
c_{s}\left(\left.\boldsymbol{u}^{\mathrm{tan}}\right|_{r=R}, \boldsymbol{U}_{n m}\right) \boldsymbol{e}_{n+1} \\
-c_{p}\left(\left.u^{r}\right|_{r=R}, Y_{n m}\right) \boldsymbol{e}_{n+1}
\end{array}\right], \quad \boldsymbol{\Psi}_{n m}(0)=0
\end{aligned}
$$

The finite element method is obtained by approximating the weak form (3.11)-(3.15) in a finite-dimensional subspace $\mathcal{V}^{h} \subset \mathcal{V}$. The domain $\Omega$ is discretized into a finite number of elements, and each element is associated with a finite number of nodes. Then $\boldsymbol{u}$ and $\boldsymbol{w}$ are approximated by

$$
\begin{aligned}
\boldsymbol{u}^{h}(\boldsymbol{x}, t) & =\sum_{i \in \eta} z_{i}(t) \boldsymbol{\Theta}_{i}(\boldsymbol{x}), \\
\boldsymbol{w}^{h}(\boldsymbol{x}, t) & =\sum_{i \in \eta} w_{i} \boldsymbol{\Theta}_{i}(\boldsymbol{x}) .
\end{aligned}
$$

Here $\eta$ is the set of indices representing the degrees of freedom for $\boldsymbol{u}^{h}, z_{i}(t)$ and $w_{i}$ are coefficients, and $\boldsymbol{\Theta}_{i}(\boldsymbol{x})$ are suitable shape functions associated with node $i$. We denote by $\beta \subset \eta$ the set of indices representing degrees of freedom corresponding to nodes on $\mathcal{B}$. Next, we substitute (3.16) and (3.17) in (3.11)-(3.15), with the sums over $n$ truncated at some finite value $N$, and require the resulting equations to hold for all values of $w_{i}$. This yields the finite element matrix form of the problem for the vector of unknowns $\boldsymbol{z}(t)=\left\{z_{i}(t)\right\}$ :

$$
\begin{aligned}
& M \ddot{z}+C \dot{z}+K z=\tilde{f} \\
& \dot{\boldsymbol{\psi}}_{n m}=\frac{1}{R} \boldsymbol{A}_{n} \boldsymbol{\psi}_{n m}+\sum_{i \in \beta} z_{i}\left(\left.\boldsymbol{\Theta}_{i}^{\tan }\right|_{r=R}, \boldsymbol{V}_{n m}\right) \boldsymbol{e}_{n}, \quad \boldsymbol{\psi}_{n m}(0)=0, \\
& \dot{\Psi}_{n m}=\frac{1}{R} \boldsymbol{B}_{n} \boldsymbol{\Psi}_{n m}+\sum_{i \in \beta} z_{i}\left[\begin{array}{c}
c_{s}\left(\left.\Theta_{i}^{\tan }\right|_{r=R}, \boldsymbol{U}_{n m}\right) \boldsymbol{e}_{n+1} \\
-c_{p}\left(\left.\Theta_{i}^{r}\right|_{r=R}, Y_{n m}\right) \boldsymbol{e}_{n+1}
\end{array}\right], \quad \Psi_{n m}(0)=0 \\
& \boldsymbol{z}(0)=\boldsymbol{z}_{0} \\
& \dot{z}(0)=\dot{z}_{0} \text {. }
\end{aligned}
$$

The matrices $\boldsymbol{M}=\{M(i, j)\}, \boldsymbol{C}=\{C(i, j)\}$, and $\boldsymbol{K}=\{K(i, j)\}$ are defined by

$$
\left.\left.M(i, j)=\left(\rho \boldsymbol{\Theta}_{i}, \boldsymbol{\Theta}_{j}\right), \quad C(i, j)=\mathcal{C}\left[\boldsymbol{\Theta}_{i}, \boldsymbol{\Theta}_{j}\right)\right], \quad K(i, j)=\mathcal{A}\left[\boldsymbol{\Theta}_{i}, \boldsymbol{\Theta}_{j}\right)\right]
$$


The vectors $\tilde{\boldsymbol{f}}=\{\tilde{f}(i)\}, \boldsymbol{z}_{0}=\left\{z_{0}(i)\right\}$, and $\dot{\boldsymbol{z}}_{0}=\left\{\dot{z}_{0}(i)\right\}$ are defined by

$$
\begin{gathered}
\tilde{f}(i)=\left(\boldsymbol{\Theta}_{i}, f\right)+\frac{2 \mu+\lambda}{R^{2}} \sum_{n \geq 0}^{N} \sum_{|m| \leq n} \boldsymbol{b}_{n} \cdot \boldsymbol{\Psi}_{n m}\left(\Theta_{i}^{r}, Y_{n m}\right)_{\mathcal{B}} \\
-\frac{\mu}{R^{2}} \sum_{n \geq 1}^{N} \sum_{|m| \leq n}\left\{\boldsymbol{d}_{n} \cdot \boldsymbol{\psi}_{n m}\left(\boldsymbol{\Theta}_{i}^{\mathrm{tan}}, \boldsymbol{V}_{n m}\right)_{\mathcal{B}}+\boldsymbol{a}_{n} \cdot \boldsymbol{\Psi}_{n m}\left(\boldsymbol{\Theta}_{i}^{\mathrm{tan}}, \boldsymbol{U}_{n m}\right)_{\mathcal{B}}\right\}, \\
z_{0}(i)=\left(\boldsymbol{\Theta}_{i}, \boldsymbol{u}_{0}\right), \quad \dot{z}_{0}(i)=\left(\boldsymbol{\Theta}_{i}, \boldsymbol{v}_{0}\right) .
\end{gathered}
$$

The quantities $\boldsymbol{z}, \dot{\boldsymbol{z}}$, and $\ddot{\boldsymbol{z}}$ are the displacement, the velocity, and the accelaration vectors, respectively. $\boldsymbol{M}$ is the mass matrix, $\boldsymbol{K}$ is the stiffness matrix, and $\boldsymbol{C}$ is a damping term. The matrix $\boldsymbol{C}$ is almost empty, since only terms on the artificial boundary yield nonzero entries. For the numerical integration of (3.18) one can use any suitable time-marching scheme, such as the explicit central difference method. Then the solutions of (3.19) and (3.20) are computed concurrently; here we recommend an implicit method, such as the trapezoidal rule, since all the eigenvalues of the matrices $\boldsymbol{A}_{n}$ and $\boldsymbol{B}_{n}$ lie in the left half of the complex plane - see sections 4 and 5. Further implementation details on the combination of the finite element method with nonreflecting boundary conditions for the scalar wave equation can be found in [26].

\section{Finite Difference Method}

Instead of using the finite element method, we can use the finite difference method to solve (2.4)-(2.8). We shall now describe how to do this, choosing $\rho(\boldsymbol{x})=1$ in $\Omega$ for simplicity. We opt for the finite difference method of Kelly et al. [27], which is a standard explicit time-marching method for the simulation of elastic waves. Then the elastic wave equation (2.4) is discretized both in time and in space at $(\boldsymbol{x}, t)$ using second-order accurate centered finite differences.

At $\mathcal{B}$ we use the boundary condition (2.6) to advance the numerical solution from time $t_{k}$ to time $t_{k+1}=t_{k}+\Delta t$. The right side of (2.6) involves infinite sums, which are truncated at a finite value $N$. It requires the values of $\boldsymbol{\psi}_{n m}(t)$ and $\boldsymbol{\Psi}_{n m}(t)$ at time $t_{k}$. These are computed concurrently with the solution inside $\Omega$, using the linear ordinary differential equations (2.7) and (2.8). We apply (2.6) at $t=t_{k}$ and $r=R$, and approximate both time and tangential derivatives by centered second order accurate finite differences. The radial derivatives in (2.6) are approximated by one-sided second order accurate finite differences. These are not evaluated at $t=t_{k}$, but instead at $t_{k-1}$ and $t_{k+1}$, since their evaluation at $t_{k}$ would lead to an unstable finite difference scheme. This instability is not particular to elastic waves. It also arises with centered finite difference approximations of the one-dimensional scalar wave equation $u_{t t}-u_{x x}=0$ together with the nonreflecting boundary condition $u_{t}+u_{x}=0$.

We now describe the finite difference approximation used at the boundary for the radial 
component of the nonreflecting boundary condition. The inner product of (2.6) with $\hat{\boldsymbol{r}}$ yields

$$
\frac{\partial u^{r}}{\partial r}+\frac{u^{r}}{R}+\frac{1}{c_{p}} \frac{\partial u^{r}}{\partial t}+\frac{c_{p}-c_{s}}{c_{p}} \nabla \cdot \boldsymbol{u}^{t a n}=\frac{1}{R^{2}} \sum_{n \geq 0}^{N} \sum_{|m| \leq n} \boldsymbol{b}_{n} \cdot \Psi_{n m} Y_{n m},
$$

which we rewrite as

$$
\frac{1}{R}\left(\frac{\partial}{\partial r}+\frac{1}{c_{p}} \frac{\partial}{\partial t}\right)\left[r u^{r}\right]=g, \quad r=R .
$$

Here $g=g(\vartheta, \phi, t)$ contains the remaining tangential derivatives of $\boldsymbol{u}^{\text {tan }}$ and the sum over $n$. We now let $U^{k}$ denote the numerical solution for the radial displacement $u^{r}$ at time $t_{k}$, and $g^{k}$ the numerical approximation of $g$ at time $t_{k}$; both $U^{k}$ and $g^{k}$ are known. Next, let $r_{\ell}=R$ denote the $\ell$-th grid point in the radial direction. Hence, $r_{\ell-1}=R-\Delta r$ and $r_{\ell-2}=R-2 \Delta r$. As mentioned above, we approximate the time derivative by centered finite differences about $t_{k}$. The radial derivative at $r_{\ell}=R$ and $t=t_{k}$ is approximated by averaging the one-sided finite differences evaluated at $t_{k-1}$ and $t_{k+1}$. This yields the following finite difference update for the radial displacement $U_{\ell}^{k+1}$ at $\mathcal{B}$ :

$$
\begin{aligned}
U_{\ell}^{k+1}= & {\left[\left(\frac{1}{2 c_{p} \Delta t}-\frac{3}{4 \Delta r}\right) U_{\ell}^{k}+\left(\frac{r_{\ell-1}}{R \Delta r}\right)\left(U_{\ell-1}^{k+1}+U_{\ell-1}^{k-1}\right)\right.} \\
& \left.-\left(\frac{r_{\ell-2}}{4 R \Delta r}\right)\left(U_{\ell-2}^{k+1}+U_{\ell-2}^{k-1}\right)+g^{k}\right] /\left[\frac{1}{2 c_{p} \Delta t}+\frac{3}{4 \Delta r}\right] .
\end{aligned}
$$

The discretization of the tangential components of (2.6) parallels that described above for the radial component and leads to similar finite difference formulas.

To solve (2.7) and (2.8) numerically, we opt for the trapezoidal rule, because the eigenvalues of the matrices $\boldsymbol{A}_{n}$ and $\boldsymbol{B}_{n}$ lie in the left half of the complex plane (see section 5). Since the trapezoidal rule is unconditionally stable, there is no restriction on the time-step in the integration of (2.7) and (2.8). The trapezoidal rule approximation of (2.7) is

$$
\left(I-\frac{\Delta t}{2 R} \boldsymbol{A}_{n}\right) \boldsymbol{\psi}_{n m}^{k+1}=\left(I+\frac{\Delta t}{2 R} \boldsymbol{A}_{n}\right) \boldsymbol{\Psi}_{n m}^{k}+\frac{\Delta t}{2}\left[\left(\boldsymbol{u}^{k}+\left.\boldsymbol{u}^{k+1}\right|_{r=R}, \boldsymbol{V}_{n m}\right)\right] \boldsymbol{e}_{n}
$$

The trapezoidal rule approximation of $(2.8)$ is

$$
\begin{aligned}
\left(I-\frac{\Delta t}{2 R} \boldsymbol{B}_{n}\right) \boldsymbol{\Psi}_{n m}^{k+1} & =\left(I+\frac{\Delta t}{2 R} \boldsymbol{B}_{n}\right) \boldsymbol{\Psi}_{n m}^{k} \\
& +\frac{\Delta t}{2}\left[\begin{array}{c}
c_{s}\left(\boldsymbol{u}^{k}+\left.\boldsymbol{u}^{k+1}\right|_{r=R}, \boldsymbol{U}_{n m}\right) \boldsymbol{e}_{n+1} \\
-c_{p}\left(\left.\hat{\boldsymbol{r}} \cdot\left(\boldsymbol{u}^{k+1}+\boldsymbol{u}^{k}\right)\right|_{r=R}, Y_{n m}\right) \boldsymbol{e}_{n+1}
\end{array}\right]
\end{aligned}
$$

The inner products in (2.7) and (2.8) are computed over the sphere $r=R$ using the fourth order Simpson rule. The work required in solving the linear systems (4.4) and (4.5) is negligible, because the matrices involved are very small; furthermore they remain constant in time if $\Delta t$ remains constant. 
The complete algorithm proceeds as follows:

0 . Initialize $\boldsymbol{u}$ at $t_{0}$ and $t_{1}$, and set $\boldsymbol{\psi}_{n m}=0$ and $\boldsymbol{\Psi}_{n m}=0$ at $t_{0}$ and $t_{1}$.

1. Compute $\boldsymbol{u}^{k+1}$ at $t_{k+1}=t_{k}+\Delta t$ at all inner points of $\Omega$ using the difference form of (2.4).

2. Compute $\boldsymbol{u}^{k+1}$ at $t_{k+1}$ and $r=R$ using (4.3) for $u^{r}$ and two similar equations, obtained from (2.6) applied at $r=R$ and $t=t_{k}$, for $\boldsymbol{u}^{\mathrm{tan}}$.

3. Compute $\boldsymbol{\psi}_{n m}^{k+1}$ and $\boldsymbol{\Psi}_{n m}^{k+1}$ at $t_{k+1}$ using (4.4) and (4.5), respectively, and return to 1 .

Most of the work involved in applying the boundary condition results from computing the inner products over $\mathcal{B}$ on the right side of (2.6). To compute the inner products it is not necessary to compute $O\left(N^{2}\right)$ inner products over the entire sphere. Indeed, since the spherical harmonics $\boldsymbol{U}_{n m}, \boldsymbol{V}_{n m}$, and $Y_{n m}$ separate in $\theta$ and $\phi$, it is sufficient to compute $O(N)$ inner products with $\cos (m \phi)$ and $\sin (m \phi)$ over the sphere, and then to compute $O\left(N^{2}\right)$ onedimensional inner products in $\theta$ over $[0, \pi]$. The same method can be used to calculate the sums over $n$ and $m$ on the right of (2.7) and (2.8). In all our computations we have found $N \leq 25$ to be sufficient. If very large values of $N$ were needed, the work and storage required could be reduced by an order of magnitude by combining the fast discrete polynomial transform of Driscoll, Healy, and Rockmore [28] with the recent work of Alpert, Greengard, and Hagstrom [29] on the approximation of boundary integral kernels - see also Hagstrom [30].

\section{$5 \quad$ Stability}

When used in computation, the boundary condition is approximated numerically. This introduces both discretization errors and rounding errors, which could lead to numerical instability. We shall now discuss the stability of the first-order systems of ordinary differential equations (2.7) and (2.8), which are used to compute the auxilliary quantities $\boldsymbol{\psi}_{n m}(t)$ and $\Psi_{n m}(t)$.

The stability of the ordinary differential equation (2.7) is determined by the eigenvalues of $\boldsymbol{A}_{n}$. In [3] we showed that the eigenvalues of $\boldsymbol{A}_{n}$, here scaled by $c_{s}$, strictly lie in the left half of the complex plane and that the differential equation (2.7) is asymptotically stable. In fact, as $n$ increases the maximal real part of the eigenvalues of $\boldsymbol{A}_{n}$ moves farther away from the imaginary axis. This results in a stronger obliteration of the past for higher Fourier modes of the displacement on $\mathcal{B}$.

The stability of the ordinary differential equation (2.8) is determined by the eigenvalues of $\boldsymbol{B}_{n}$. For $n \geq 1$, the matrix $\boldsymbol{B}_{n}$ has a zero eigenvalue with associated eigenvector $\boldsymbol{v}=\{\boldsymbol{v}(j)\}$, $j=1, \ldots, 2(n+1)$,

$$
\boldsymbol{v}(j)= \begin{cases}-1 & \text { if } j=n+1, \\ \sqrt{n /(n+1)} & \text { if } j=2(n+1), \\ 0 & \text { otherwise }\end{cases}
$$


Moreover, there exists a second vector $\boldsymbol{w}$, linearly independent of $\boldsymbol{v}$, such that $\boldsymbol{B}_{n} \boldsymbol{w}=\boldsymbol{v}$. It is

$$
\boldsymbol{w}(j)= \begin{cases}-1 / c_{s} & \text { if } j=n, \\ 1 / c_{s} & \text { if } j=n+1, \\ \sqrt{n /(n+1)} / c_{p} & \text { if } j=2 n+1, \\ -\sqrt{n /(n+1)} / c_{p} & \text { if } j=2(n+1), \\ 0 & \text { otherwise. }\end{cases}
$$

Thus $\left(\boldsymbol{B}_{n}\right)^{2} \boldsymbol{w}=0$ and the two-dimensional subspace $V=\{\boldsymbol{v}, \boldsymbol{w}\}$, spanned by $\boldsymbol{v}$ and $\boldsymbol{w}$, is invariant under $\boldsymbol{B}_{n}$. Moreover, the forcing term on the right of (2.8) is clearly orthogonal to $\boldsymbol{v}$ and $\boldsymbol{w}$. Therefore, the projection of $\boldsymbol{\Psi}_{n m}(t)$ on $V$ is identically zero for all time, that is $\boldsymbol{v} \cdot \boldsymbol{\Psi}_{n m}(t) \equiv 0$ and $\boldsymbol{w} \cdot \boldsymbol{\Psi}_{n m}(t) \equiv 0$, and does not play any role in (2.8).

Because of rounding errors the $2 \times 2$ Jordan block associated with the zero eigenvalue could possibly lead to spurious linear growth in time. However, a direct calculation reveals that $\boldsymbol{a}_{n}$ and $\boldsymbol{b}_{n}$ are orthogonal to both $\boldsymbol{v}$ and $\boldsymbol{w}$. Therefore, the component of $\boldsymbol{\Psi}_{n m}(t)$ in $V$ plays no role in the boundary condition (2.6).

Next, we shall show how to remove the superfluous invariant subspace $V$ and obtain an equivalent formulation, which is asymptotically stable for all $n$. To do so, we seek a matrix representation of the projection on $V^{\perp}$, the orthogonal complement of $V$. First, we find two othonormal vectors $\boldsymbol{q}_{1}=\left\{q_{1}(j)\right\}$ and $\boldsymbol{q}_{2}=\left\{q_{2}(j)\right\}, j=1, \ldots, 2(n+1)$, which are orthogonal to both $\boldsymbol{v}$ and $\boldsymbol{w}$ :

$$
\begin{gathered}
\boldsymbol{q}_{1}(j)= \begin{cases}\left(1+\left[(n+1) c_{p}^{2}\right] /\left[n c_{s}^{2}\right]\right)^{-1 / 2} & \text { if } j=n, \\
\left(1+\left[n c_{s}^{2}\right] /\left[(n+1) c_{p}^{2}\right]\right)^{-1 / 2} & \text { if } j=2 n+1, \\
0 & \text { otherwise. }\end{cases} \\
\boldsymbol{q}_{2}(j)= \begin{cases}-\sqrt{(n+1) / n}\left(c_{p} / c_{s}\right) C & \text { if } j=n, \\
\sqrt{n /(n+1)} D & \text { if } j=n+1, \\
C & \text { if } j=2 n+1, \\
D & \text { if } j=2(n+1), \\
0 & \text { otherwise. }\end{cases}
\end{gathered}
$$

where the constants $C$ and $D$ are given by

$$
\begin{aligned}
C & =\left[1+\frac{(n+1)\left(c_{p} / c_{s}\right)^{2}}{n}+\left(\frac{n}{n+1}+1\right)\left(\frac{(n+1) c_{p}^{2}+n c_{s}^{2}}{n\left(c_{p}-c_{s}\right) c_{s}}\right)^{2}\right]^{-1 / 2} \\
D & =-\left[\frac{(n+1) c_{p}^{2}+n c_{s}^{2}}{n\left(c_{p}-c_{s}\right) c_{s}}\right] C .
\end{aligned}
$$


Next, we let $\boldsymbol{Q}_{n}$ denote the $2(n+1) \times 2 n$ matrix, whose columns form an orthonormal basis of $V^{\perp}$ :

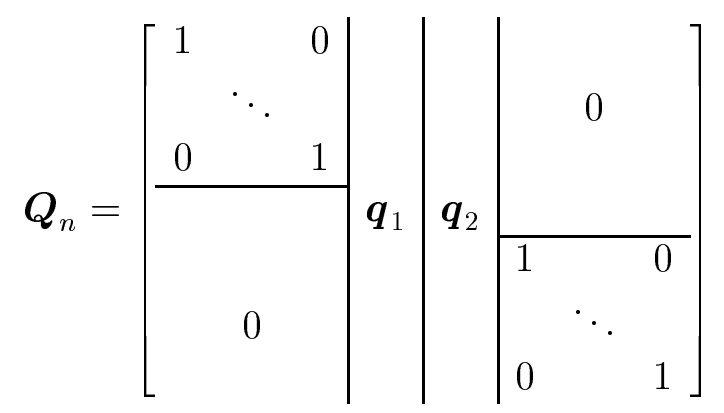

The upper left and the lower right blocks of $\boldsymbol{Q}_{n}$ correspond to two $(n-1) \times(n-1)$ identity matrices.

We now let

$$
\tilde{\boldsymbol{B}}_{n}=\boldsymbol{Q}_{n}^{\top} \boldsymbol{B}_{n} \boldsymbol{Q}_{n}, \quad \tilde{\boldsymbol{\Psi}}_{n m}(t)=\boldsymbol{Q}_{n}^{\top} \boldsymbol{\Psi}_{n m}(t),
$$

and multiply (2.8) by $\boldsymbol{Q}_{n}^{\top}$ from the left to obtain the equivalent linear system of ordinary differential equations

$$
\frac{d}{d t} \tilde{\boldsymbol{\Psi}}_{n m}=\frac{1}{R} \tilde{\boldsymbol{B}}_{n} \tilde{\boldsymbol{\Psi}}_{n m}+\left[\begin{array}{c}
c_{s}\left(\left.\boldsymbol{u}^{\mathrm{tan}}\right|_{r=R}, \boldsymbol{U}_{n m}\right) \boldsymbol{e}_{n} \\
-c_{p}\left(\left.u^{r}\right|_{r=R}, Y_{n m}\right) \boldsymbol{e}_{n}
\end{array}\right], \quad \tilde{\boldsymbol{\Psi}}_{n m}(0)=0 .
$$

We have calculated the eigenvalues of $\tilde{\boldsymbol{B}}_{n}$ and they are shown for $n=10$ in the left frame of Figure 1. We observe that all the eigenvalues of $\tilde{\boldsymbol{B}}_{n}$, here for $n=10$, have strictly negative imaginary parts. Furthermore, as shown in the right frame of Figure 1, they tend to move farther away from the imaginary axis with increasing $n$. Again this results in a stronger obliteration of the past for higher Fourier modes of the displacement on $\mathcal{B}$. We verified numerically that different $c_{p} / c_{s}$ ratios always lead to the same conclusion. Thus, by removing the two-dimensional subspace $V$ associated with the zero eigenvalue, we have obtained the equivalent differential equation (5.9), which is asymptotically stable for all time. With

$$
\tilde{\boldsymbol{a}}_{n}=\boldsymbol{Q}_{n}^{\top} \boldsymbol{a}_{n}, \quad \tilde{\boldsymbol{b}}_{n}=\boldsymbol{Q}_{n}^{\top} \boldsymbol{b}_{n},
$$

the exact nonreflecting boundary condition is now given by (2.6) but with $\boldsymbol{a}_{n}, \boldsymbol{b}_{n}$, and $\boldsymbol{\Psi}_{n m}(t)$ replaced by $\tilde{\boldsymbol{a}}_{n}, \tilde{\boldsymbol{b}}_{n}$, and $\tilde{\boldsymbol{\Psi}}_{n m}(t)$, respectively. We summarize the main results of this section in the following proposition.

Proposition 1 For $n \geq 1$, let $\boldsymbol{v}, \boldsymbol{w}$, and $\boldsymbol{Q}_{n}$ be defined by (5.1), (5.2), and (5.7). Then

1. $\boldsymbol{B}_{n} \boldsymbol{v}=0, \boldsymbol{B}_{n} \boldsymbol{w}=\boldsymbol{v}$, and $\boldsymbol{Q}_{n}^{\top}$ is an orthogonal projection on $V^{\perp}$, where $V=$ $\operatorname{span}\{\boldsymbol{v}, \boldsymbol{w}\}$,

2. $\boldsymbol{v} \cdot \boldsymbol{\Psi}_{n m}(t) \equiv 0$ and $\boldsymbol{w} \cdot \Psi_{n m}(t) \equiv 0$,

3. $\boldsymbol{a}_{n} \cdot \boldsymbol{v}=\boldsymbol{b}_{n} \cdot \boldsymbol{v}=\boldsymbol{a}_{n} \cdot \boldsymbol{w}=\boldsymbol{b}_{n} \cdot \boldsymbol{w}=0$, 
4. Numerical calculations indicate that the eigenvalues of $\tilde{\boldsymbol{B}}_{n}=\boldsymbol{Q}_{n}^{\top} \boldsymbol{B}_{n} \boldsymbol{Q}_{n}$ have strictly negative real parts.

Both formulations (2.8) and (5.9) were implemented and they lead to identical results.

Remark:

For large values of $n$, the problem of computing the eigenvalues of either $\boldsymbol{A}_{n}, \boldsymbol{B}_{n}$, or $\tilde{\boldsymbol{B}}_{n}$ is extremely ill-conditioned, so that meaningful results cannot be obtained even in double precision. This fact is of no consequence for the use of the nonreflecting boundary condition, because these eigenvalues are never needed - for further details refer to ([3], Section 6).

\section{Numerical results}

We shall now combine the finite-difference method with the nonreflecting boundary condition (2.6), as described in section 4, to evaluate its accuracy and convergence properties. First, we shall consider a model problem, for which the exact solution is known. It is just to find the field of a transient point dipole source in a homogeneous medium. Second, we shall present computations for a standard test problem: scattering from a spherical cavity. Both problems are symmetric about the $z$-axis and therefore $\boldsymbol{u}$ is independent of $\phi$. Moreover, the $\phi$-component of $\boldsymbol{u}$ decouples from the $r$ - and $\vartheta$-components of $\boldsymbol{u}$ in (2.1) and (2.6); it is governed by a scalar wave equation. In [3] we have presented examples which show the accuracy of this method for the scalar wave equation, and we have discussed storage requirements and other computational issues. To avoid repetition, we shall set the $\phi$-component of $\boldsymbol{u}$ to zero and focus on the main new feature of the nonreflecting boundary condition for elastic waves, namely the coupling of compression and shear modes through $\Psi_{n m}(t)$ in $(2.6)$ and (2.8).

\subsection{Model problem}

We consider the displacement wave field produced by a time dependent dipole source, $P(t)$, located at $S_{1}=\left(0,0, z_{0}\right)$. If $z_{0}=0$ the exact solution is

$$
\begin{aligned}
\boldsymbol{u}(r, \vartheta, t)= & {\left[\frac{\varphi_{p}^{\prime \prime}}{c_{p}^{2}}+\frac{2 \varphi_{p}^{\prime}}{c_{p} r}+\frac{2 \varphi_{p}}{r^{2}}+\sqrt{2}\left(\frac{\varphi_{s}^{\prime}}{c_{s} r}+\frac{\varphi_{s}}{r^{2}}\right)\right] \frac{Y_{10}(\vartheta)}{r} \hat{\boldsymbol{r}} } \\
- & {\left[\frac{\varphi_{s}^{\prime \prime}}{c_{s}^{2}}+\frac{\varphi_{s}^{\prime}}{c_{s} r}+\frac{\varphi_{s}}{r^{2}}+\sqrt{2}\left(\frac{\varphi_{p}^{\prime}}{c_{p} r}+\frac{\varphi_{p}}{r^{2}}\right)\right] \frac{\boldsymbol{U}_{10}(\vartheta)}{r}, }
\end{aligned}
$$

where

$$
\varphi_{p}=P\left(t-r / c_{p}\right), \quad \varphi_{s}=P\left(t-r / c_{s}\right),
$$

and $Y_{10}$ and $\boldsymbol{U}_{10}$ are defined by (2.10) and (2.11), with $n=1$ and $m=0$. Next, we shift the source by a distance $z_{0}$ from the origin: now all Fourier modes of the solution $\boldsymbol{u}_{n m}$ are 
nonzero. The time dependence of the source, shown in Fig. 2, is a Gaussian pulse centered at $t=t_{0}$ :

$$
P(t)= \begin{cases}0 & t<0, \\ e^{-\left(t-t_{0}\right)^{2} / \sigma^{2}} & 0 \leq t \leq 2 t_{0} \\ 0 & t>2 t_{0}\end{cases}
$$

We choose $t_{0}=1$ and set $\sigma$ so that $P(t)$ is equal to $10^{-16}$ at $t=0$ and $t=2 t_{0}$.

We impose the exact displacement at $r=r_{0}$ and compute its propagation outwards up to the artificial boundary $r=R$. Because of the inherent symmetry, the computational domain $\Omega$ can be reduced to the two-dimensional region $r_{0} \leq r \leq R, 0 \leq \vartheta \leq \pi$, shown in Fig. 3. Inside $\Omega$ we use polar coordinates and a uniform mesh in $r$ and $\vartheta$. We non-dimensionalize the distance by the diameter $2 r_{0}$ of the inner sphere and time by $2 r_{0} / c_{p}$, the travel time of a compression wave across the inner sphere. Thus, $r_{0}=0.5, c_{p}=1$. Furthermore, we set $R=1$ and $c_{s}=1 / \sqrt{3}$.

We shall compare the numerical solution using (2.6), where the sums are truncated at $N$, with that obtained using the 'viscous' boundary condition of Lysmer and Kuhlemeyer [7],

$$
\begin{aligned}
& T^{r r}+c_{p} \frac{\partial u^{r}}{\partial t}=0 \\
& T^{r \vartheta}+c_{s} \frac{\partial u^{\vartheta}}{\partial t}=0,
\end{aligned}
$$

in which $T^{r r}$ and $T^{r \vartheta}$ are the normal and shear stress, respectively. We denote the former by $\operatorname{NBC}(N)$, where $N$ indicates the upper limit in the sums, and the latter by L-K. The boundary condition (2.6) is implemented as described in section 4 , albeit $\boldsymbol{\psi}_{n m}(t) \equiv 0$ because $u^{\phi}$ is identically zero.

In figure 4 we check the accuracy and convergence rate of our numerical method. In the left frame the maximal error in the $L_{2}$-norm over the time interval $[0,10]$ is shown versus the mesh parameter $\Delta r$, for the following sequence of meshes: $20 \times 120,30 \times 180,40 \times 240$, $60 \times 360,80 \times 480$, and $100 \times 600$. We observe the expected second order convergence rate of the full scheme using $\mathrm{NBC}(25)$ as the mesh is refined. This indicates that setting $N=25$ ensures that the error introduced at the artificial boundary is smaller than that of the numerical scheme. However, the error in the numerical solution obtained with L-K barely decreases as the mesh is refined, indicating that the error introduced by using L-K dominates the computation. Indeed, the numerical solution does not converge to the solution of the original problem, but instead converges to the solution of a different problem with $\mathrm{L}-\mathrm{K}$ imposed at $\mathcal{B}$. To reduce the amount of spurious reflection at $\mathcal{B}$ one would need to increase the size of $\Omega$. In contrast, for the exact boundary condition, $\operatorname{NBC}(N), N$ can be chosen large enough to reduce the error introduced at $\mathcal{B}$ below the discretization error of the numerical method inside $\Omega$, without moving the artificial boundary farther away from the scatterer. In the right frame of figure 4 we follow the evolution of the total errors in the 2-norm over $\Omega,\left\|\boldsymbol{u}_{\text {exact }}(., t)-\boldsymbol{u}_{\text {num }}(., t)\right\|_{2}$, which result from the use of L-K and NBC(25). We observe that $\mathrm{NBC}(25)$ leads to an additional reduction in the error of two orders of 
magnitude. Moreover, the error in the numerical solution obtained with $\mathrm{NBC}(25)$ decays much faster with increasing time once the transient wave field has left $\Omega$. This indicates that using the nonreflecting boundary condition may be useful even in calculations where the transient field is of no interest, since the numerical solution may reach the final state much faster.

Next, we compare the numerical solutions, obtained on the finest mesh using L-K and $\mathrm{NBC}(25)$, with the exact solution at two different locations inside $\Omega$ at $r=0.75: P_{1}$ $\left(\theta=30^{\circ}\right)$ and $Q_{1}\left(\theta=150^{\circ}\right)$. The inner and outer radii remain at their current locations $r_{0}=0.5$ and $R=1$. In figure 5 , the $\vartheta$-component of the displacement $u^{\vartheta}$ is shown at the first location $P_{1}$. The numerical solution obtained with $\mathrm{NBC}(25)$ is hardly distinguishable from the exact solution. While the relative error due to the L-K boundary condition lies within 20 percent of the maximum of the exact solution at $P_{1}$, this seemingly accurate behavior is deceptive. Indeed these locally small reflections travel back into the computational domain, and contaminate the solution everywhere inside $\Omega$, in particular in regions where the solution is of lesser magnitude. To demonstrate this point, we select the next location farther away from the source at $Q_{1}$, where the displacement field is weaker. The $\vartheta$-component of the displacement at $Q_{1}$ is shown in figure 6, and again it agrees completely with the numerical solution obtained using $\mathrm{NBC}(25)$. The solution obtained using L-K agrees with the exact solution up to $t=3$. It then diverges from it, as the spurious reflection due to the imposition of L-K reaches this location. Since this spurious reflection is as large as the amplitude of the true solution at $Q_{1}$, the numerical solution with $\mathrm{L}-\mathrm{K}$ imposed at $\mathcal{B}$ becomes meaningless after $t=3$.

\subsection{Spherical cavity}

We shall now compute the scattered field of a radially symmetric compression wave impinging upon a spherical cavity of radius $r_{0}$ embedded in an infinite elastic medium. At the free surface of the cavity the traction is zero. The source is located nearby outside the spherical obstacle at $S_{2}=\left(0,0, z_{0}\right), z_{0}>r_{0}$, at distance $z_{0}$ from the origin. The time dependence of the point load, shown in figure 2 , is the same Gaussian pulse centered in time about $t_{0}=1$ and given by (6.4). Again, this problem is symmetric about the $z$-axis and the $\phi$-component of $\boldsymbol{u}$ is identically zero. Next, we split the total displacement field $\boldsymbol{u}$ into the incident field, $\boldsymbol{u}^{i}$, and the scattered field, $\boldsymbol{u}^{s}$, with $\boldsymbol{u}=\boldsymbol{u}^{i}+\boldsymbol{u}^{s}$. The incident field is known and can be found in [24], p. 475. Since the normal and shear stress components of the total displacement vanish at $r=r_{0}$, the stress components of the scattered field $\boldsymbol{u}^{s}$ simply equal those of the incident field, but with opposite sign: $\boldsymbol{T}\left[\boldsymbol{u}^{s}\right] \hat{\boldsymbol{r}}=-\boldsymbol{T}\left[\boldsymbol{u}^{i}\right] \hat{\boldsymbol{r}}$.

We nondimensionalize time and space as before and set $c_{p}=1, c_{s}=1 / \sqrt{3}, r_{0}=0.5$, and $z_{0}=0.6$. Hence the source $S_{2}$ is located at distance 0.1 away from the spherical cavity. Since we do not have a simple analytic expression for the time dependent scattered field, we shall use the numerical solution in the infinite domain as our reference solution; we refer to it as 
the exact solution. To compute it inside $\Omega$ we use a much larger domain which extends as far as $r=6$. This enables us to compute the solution of the initial-boundary value problem in the infinite region outside the cavity up to $t=10$. Indeed the truncation at $r=6$ will not be sensed inside $\Omega$ until $t=10.5$.

Inside $\Omega$ we use a $60 \times 360$ mesh, which we extend with constant mesh spacing $\Delta r$ up to $r=6$. In figure 7 the contour lines for the displacement $|\boldsymbol{u}|$ of the exact solution are shown at $t=5$ for $r_{0} \leq r \leq 5$. The scattered field consists of various types of waves which propagate at different speeds. The impact above the north pole immediately creates a pure compression wave moving upward, followed by a shear wave propagating away from the cavity. At the surface of the cavity, a Rayleigh wave propagates along longitudes down to the south pole, while its tail interacts in a complex pattern with the only slightly faster shear wave. The surface waves merge at the south pole at about $t=5$, as shown in figure 7 , and then pursue their journey around the cavity back to the north pole, and so forth, indefinitely.

We now compare the numerical solutions, obtained inside $\Omega$ with L-K and $\mathrm{NBC}(25)$ applied at $\mathcal{B}(R=1)$, with the exact solution at two different locations on the free surface: $P_{2}\left(\theta=30^{\circ}\right)$ and $Q_{2}\left(\theta=150^{\circ}\right)$. In figure 8, the radial displacement $u^{r}$ is shown at the first location $P_{2}$. The solution obtained using L-K agrees with the exact solution up to $t=2.5$. It then diverges from it, as the spurious reflection due to the imposition of L-K reaches this location. To demonstrate the long-time accuracy of our method, we have magnified the scale and concentrate on the second wave packet, which arrives at $P_{2}$ around $t=7$ after traveling counter-clockwise around the entire cavity. At this stage, the main part of the scattered field has left $\Omega$ and the remaining part is of much smaller magnitude. Yet even after an entire trip around the cavity, the scattered field obtained with $\mathrm{NBC}(25)$ agrees perfectly with that in the unbounded region. In contrast, the local boundary condition L-K has generated multiple spurious reflections which bounce back and forth between the sphere and the artificial boundary, and completely dominate the numerical solution at later times.

Finally, we follow the evolution of the $\vartheta$-component of the displacement at $Q_{2}$, which is located on the obstacle in the shadow region behind the cavity. Here the amplitude of the scattered field is of smaller magnitude. As depicted in figure 9, the numerical solution obtained with $\mathrm{NBC}(25)$ follows the exact solution closely and cannot be distinguished from it. In contrast, the spurious reflections introduced by L-K at the outer boundary are larger than the true solution and spoil the solution right up to the obstacle as they travel back into $\Omega$. The accuracy of the numerical solution obtained with L-K remains poor in the shadow region and does not converge to the exact solution, as the underlying mesh is refined. To study the performance of the boundary conditions as the outer boundary $\mathcal{B}$ is moved closer to the inner one, we now set $R=0.6$. The mesh size remains identical, so that the mesh now has $12 \times 360$ points. In figure 10 , the $\vartheta$-component of the displacement is again shown at $Q_{2}$ below the spherical cavity. Again, the numerical solution obtained using NBC(25) agrees with the exact solution; this demonstrates the robustness of the exact boundary condition with respect to the location of the artificial boundary. The numerical solution obtained with 
L-K agrees with the exact solution for a short time. It then strongly overshoots, completely misses the subsequent arrival of the shear wave, and slowly starts to approach zero.

\section{Conclusion}

The exact nonreflecting boundary condition (2.6) has been found to be very accurate in numerical computations. It involves only first-order derivatives of the displacement, which makes it robust and easy to use. The boundary condition fits easily into finite-difference methods and allows the artificial boundary to be brought as close as desired to the scatterer. It is easy to implement and requires little extra storage and computer time. It also fits naturally into the variational formulation of the elastic wave equation; hence it is well-suited for use with the finite element method. Although the formulation is global over the artificial boundary, it is explicit and does not require the solution of any large linear system. It only requires inner products with spherical harmonics of the displacement on the artificial boundary. Although the artificial boundary must be spherical, the boundary condition is not tied to any coordinate system, and the grid used inside $\Omega$ can be arbitrary. With the nonreflecting boundary condition the overall numerical scheme retains its optimal rate of convergence, as the error introduced at the artificial boundary can always be reduced below the discretization error due to the numerical method in the interior computational domain.

\section{Acknowledgement}

I would like to thank Bradley Alpert and Joseph B. Keller for helpful comments and suggestions.

\section{References}

[1] M. J. Grote and J. B. Keller, "Nonreflecting boundary conditions for elastic waves", SIAM J. Appl. Math., in press.

[2] M. J. Grote and J. B. Keller, "Exact nonreflecting boundary conditions for the time dependent wave equation", SIAM J. Appl. Math. 55, 280 (1995).

[3] M. J. Grote and J. B. Keller, "Nonreflecting boundary conditions for time dependent scattering", J. Comput. Phys. 127, 52 (1996).

[4] M. J. Grote and J. B. Keller, "Nonreflecting boundary conditions for Maxwell's equations", J. Comput. Phys. 139, 327 (1998).

[5] D. Givoli, Numerical Methods for Problems in Infinite Domains (Studies in Applied Mechanics 33, Elsevier, 1992). 
[6] S. V. Tsynkov, "Numerical solution of problems on unbounded domains. A review", Appl. Num. Math. 27, 465 (1998).

[7] J. Lysmer and R. L. Kuhlemeyer, "Finite dynamic model for infinite media", J. Engin. Mech. Div., Proc. Am. Soc. Civil Eng., 95, 859 (1969).

[8] R. W. Clayton and B. Engquist, "Absorbing boundary conditions for acoustic and elastic wave equations", Bull. Seis. Soc. Am. 67, 1529 (1977).

[9] B. Engquist and A. Majda, "Absorbing boundary conditions for the numerical simulation of waves", Math. Comp. 31, 629 (1977).

[10] B. Engquist and A. Majda, "Radiation boundary conditions for acoustic and elastic wave calculations", Comm. Pure and Appl. Math. 32, 313 (1979).

[11] R. L. Higdon, "Numerical absorbing boundary conditions for the wave equation", Math. Comp. 49, 65 (1987).

[12] R. L. Higdon, "Radiation boundary conditions for elastic wave propagation", SIAM J. Num. Analysis 27, 831 (1990).

[13] E.L. Lindman, "Free space boundary conditions for the time dependent wave equation", J. Comput. Phys. 18, 66 (1975).

[14] C. J. Randall, "Absorbing boundary condition for the elastic wave equation", Geophysics 53, 611 (1988).

[15] C. J. Randall, "Absorbing boundary condition for the elastic wave equation: Velocity-stress formulation", Geophysics 54, 1141 (1989).

[16] M. Israeli and S. A. Orszag, "Approximation of radiation boundary conditions", J. Comput. Phys. 41, 115 (1981).

[17] J.-P. Bérenger, "A perfectly matched layer for the absorbtion of electromagnetic waves", J. Comput. Phys. 114, 185 (1994).

[18] Y.-G. Zhang and J. Ballmann, "Two techniques for the absorbtion of elastic waves using an artificial transition layer", Wave Motion 25, 15 (1997).

[19] L. H. Howell and L. N. Trefethen, Ill-posedness of absorbing boundary conditions for migration, Geophysics 53, 593 (1988).

[20] D. Givoli and J. B. Keller, "Non-reflecting boundary conditions for elastic waves", Wave Motion 12, 261 (1990). 
[21] I. Harari and Z. Shohet, "On Non-reflecting boundary conditions in unbounded elastic solids", Comp. Meth. Appl. Mech. Eng. 163, 123 (1998).

[22] L. Ting and M. J. Miksis, "Exact boundary conditions for scattering problems", J. Acoust. Soc. Amer. 80, 1825 (1986).

[23] D. Givoli and D. Cohen, "Nonreflecting boundary conditions based on Kirchhofftype formulae", J. Comput. Phys. 117, 102 (1995).

[24] A. C. Eringen and E. S. Şuhubi, Elastodynamics, vol. II (Academic Press, New York, 1975).

[25] D. Colton and R. Kress, Inverse Acoustic and Electromagnetic Scattering Theory (Springer Verlag, New York, 1992).

[26] L. L. Thompson and R. Huan, "Implementation of exact non-reflecting boundary conditions in the finite element method for the time-dependent wave equation", Comput. Meth. Appl. Mech. Engin., in press.

[27] K. R. Kelly, R. W. Ward, S. Treitel, and R. M. Alford, "Synthetic seismograms: a finite-difference approach", Geophysics 41, 2 (1976).

[28] J. R. Driscoll, D. M. Healy, Jr., and D. N. Rockmore, "Fast discrete polynomial transforms with applications to data analysis for distance transitive graphs", SIAM J. Comput. 26, 1066 (1997).

[29] B. Alpert, L. Greengard and T. Hagstrom, "Rapid evaluation of nonreflecting boundary kernels for time-domain wave propagation", SIAM J. Numer. Anal., in press.

[30] T. Hagstrom, "Radiation boundary conditions for the numerical simulation of waves", Acta Numerica (Cambridge University Press, 1999), p. 47. 


\section{Figure captions}

Figure 1: Left: the eigenvalues of the matrix $\tilde{\boldsymbol{B}}_{n}$ for $n=10, c_{p}=1$, and $c_{s}=1 / \sqrt{3}$. Right: the maximal real part of the eigenvalues of $\tilde{\boldsymbol{B}}_{n}$ as a function of $n$.

Figure 2: The time dependence $P(t)$ of the source

Figure 3: The computational domain $\Omega$ is shown drawn to scale, with $r_{0}=0.5$ and $R=1$. The source is located at $S_{1}=(0,0,0.4)$ for the model problem and at $S_{2}=(0,0,0.6)$ for scattering from a spherical cavity.

Figure 4: Left: the maximal error in the $L_{2}$ norm over the time interval [0,15] is shown versus the mesh parameter $\Delta r$. Right: the maximal error is shown as a function of time for the finest mesh $80 \times 480$ used.

Figure 5: Model problem. The numerical solutions for $u^{\vartheta}$, computed using the boundary conditions $\mathrm{L}-\mathrm{K}$ and $\mathrm{NBC}(25)$, are compared with the exact solution at $P_{1}$.

Figure 6: Model problem. The numerical solutions for $u^{\vartheta}$, computed using the boundary conditions L-K and $\mathrm{NBC}(25)$, are compared with the exact solution at $Q_{1}$.

Figure 7: Spherical cavity. Contour lines of the exact solution are shown at $t=5$. The dotted circle in the center shows the initial location of the spherical cavity.

Figure 8: Spherical cavity. The numerical solutions for $u^{r}$, computed using the boundary conditions $\mathrm{L}-\mathrm{K}$ and $\mathrm{NBC}(25)$ at $R=1$, are compared with the exact solution at $P_{2}$.

Figure 9: Spherical cavity. The numerical solutions for $u^{\vartheta}$, computed using the boundary conditions L-K and $\mathrm{NBC}(25)$ at $R=1$, are compared with the exact solution at $Q_{2}$.

Figure 10: Spherical cavity. The numerical solutions for $u^{\vartheta}$, computed using the boundary conditions L-K and $\mathrm{NBC}(25)$ at $R=0.6$, are compared with the exact solution at $Q_{2}$. 

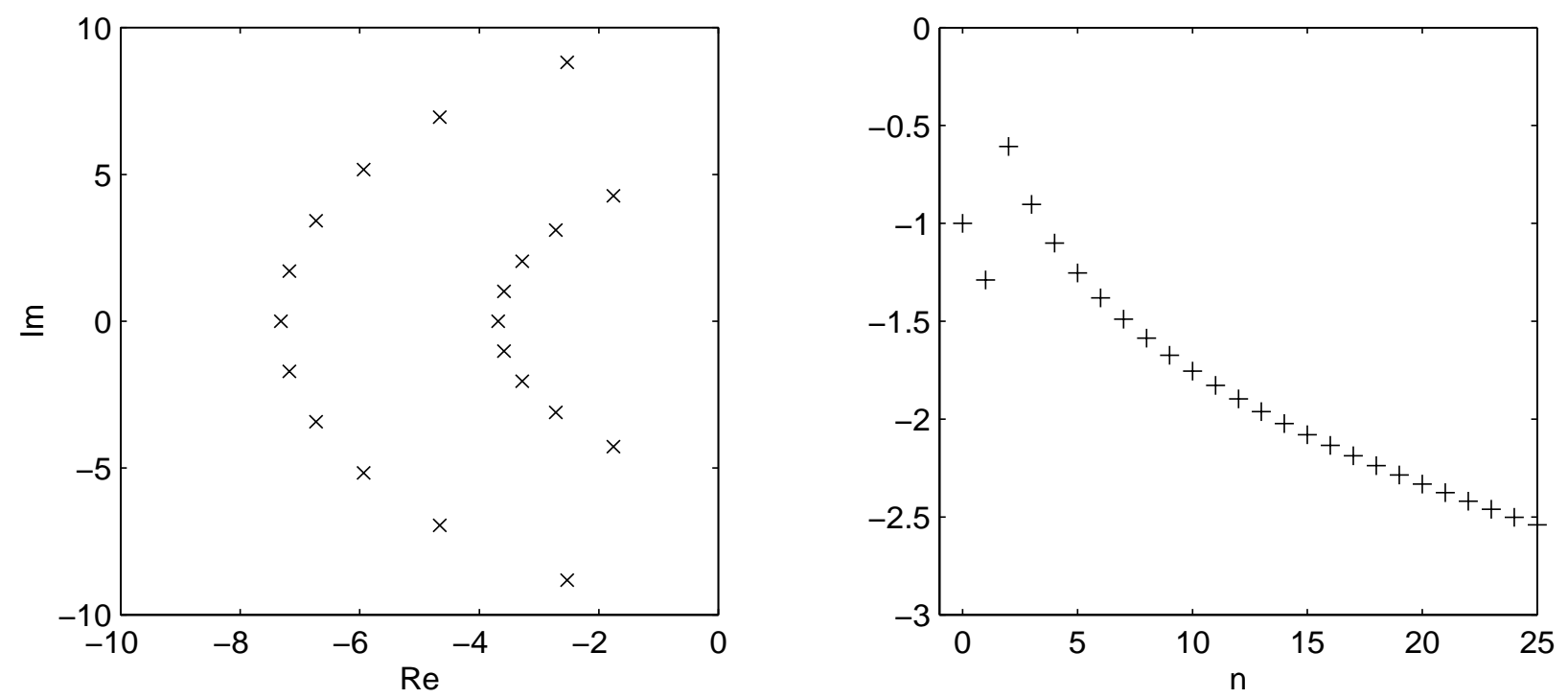

Figure 1: 


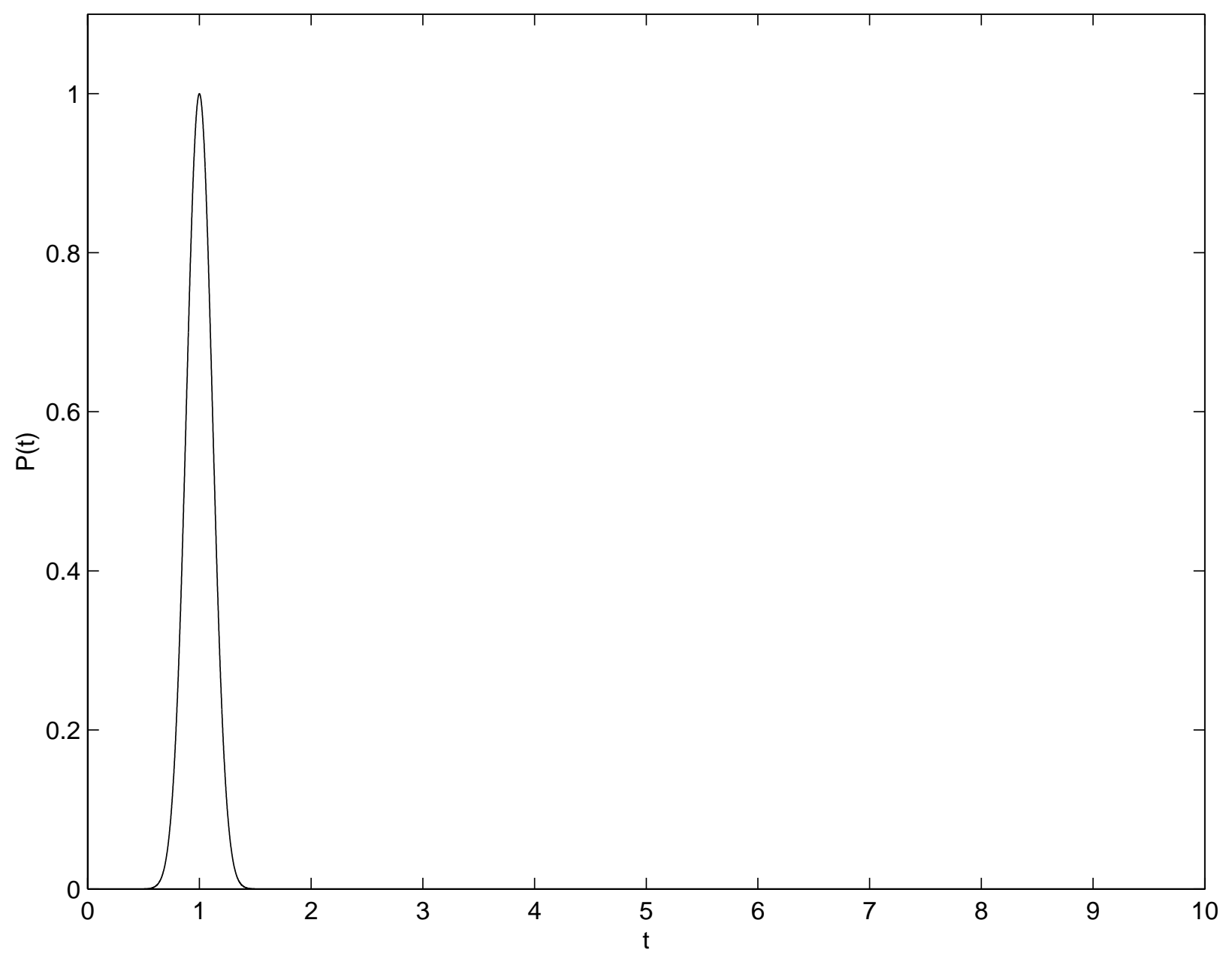

Figure 2: 


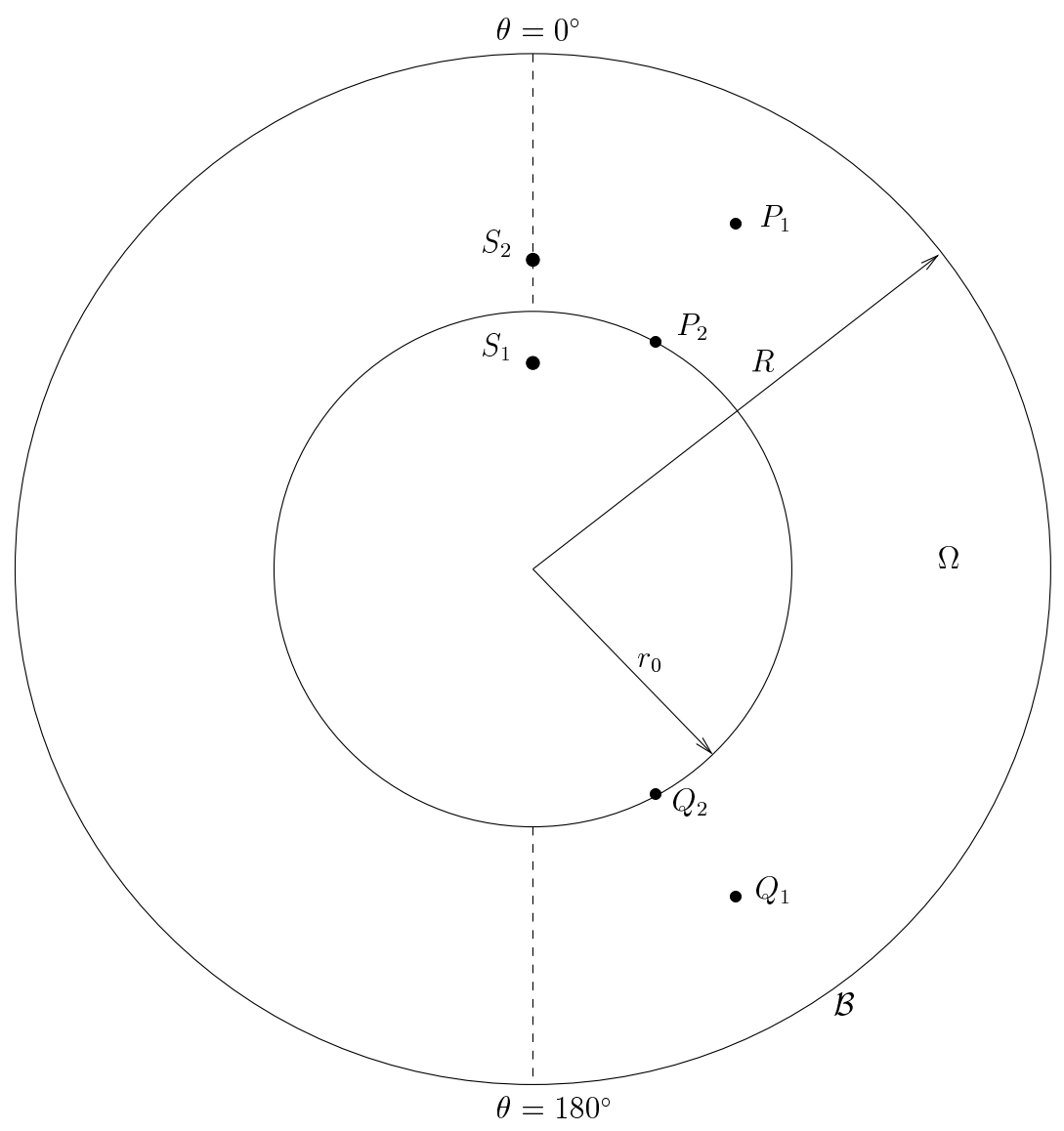

Figure 3: 

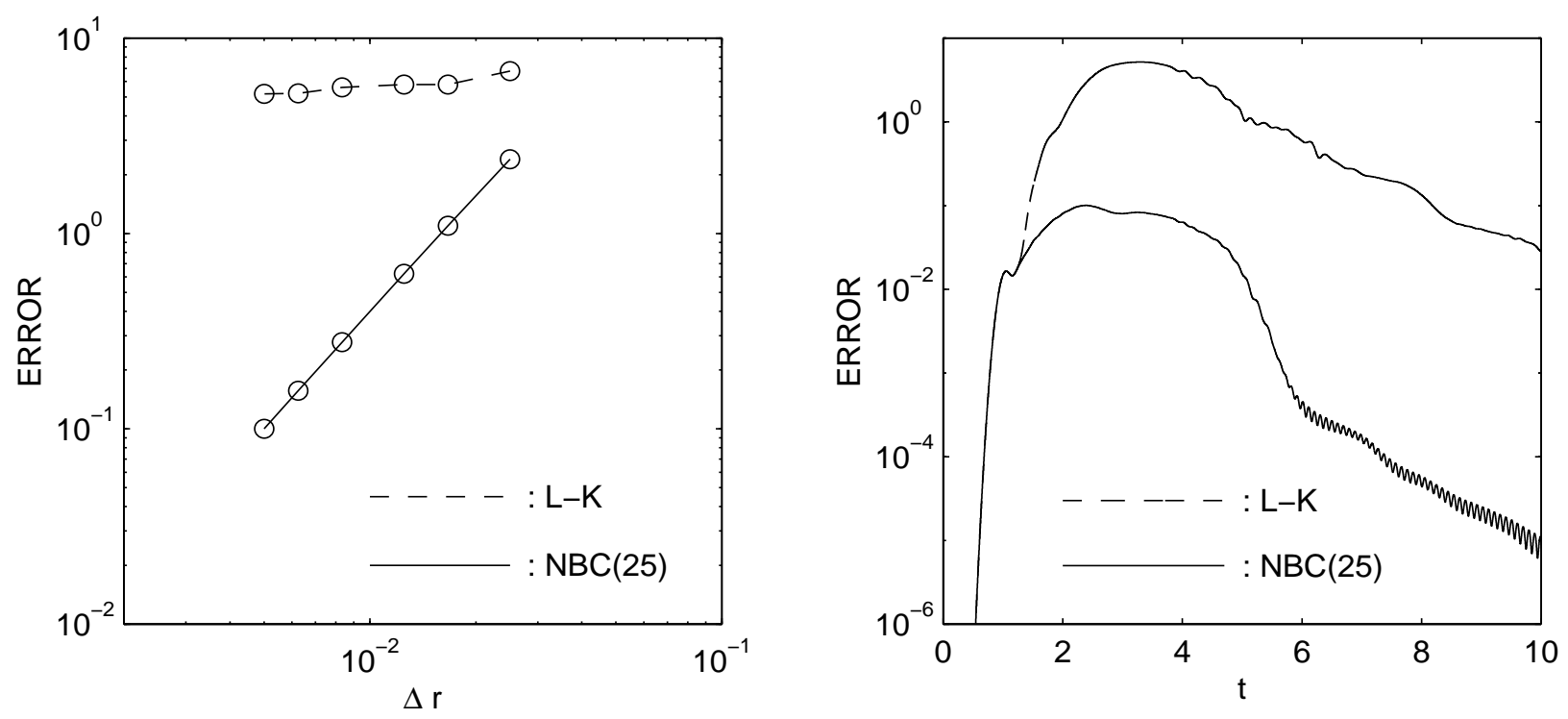

Figure 4: 


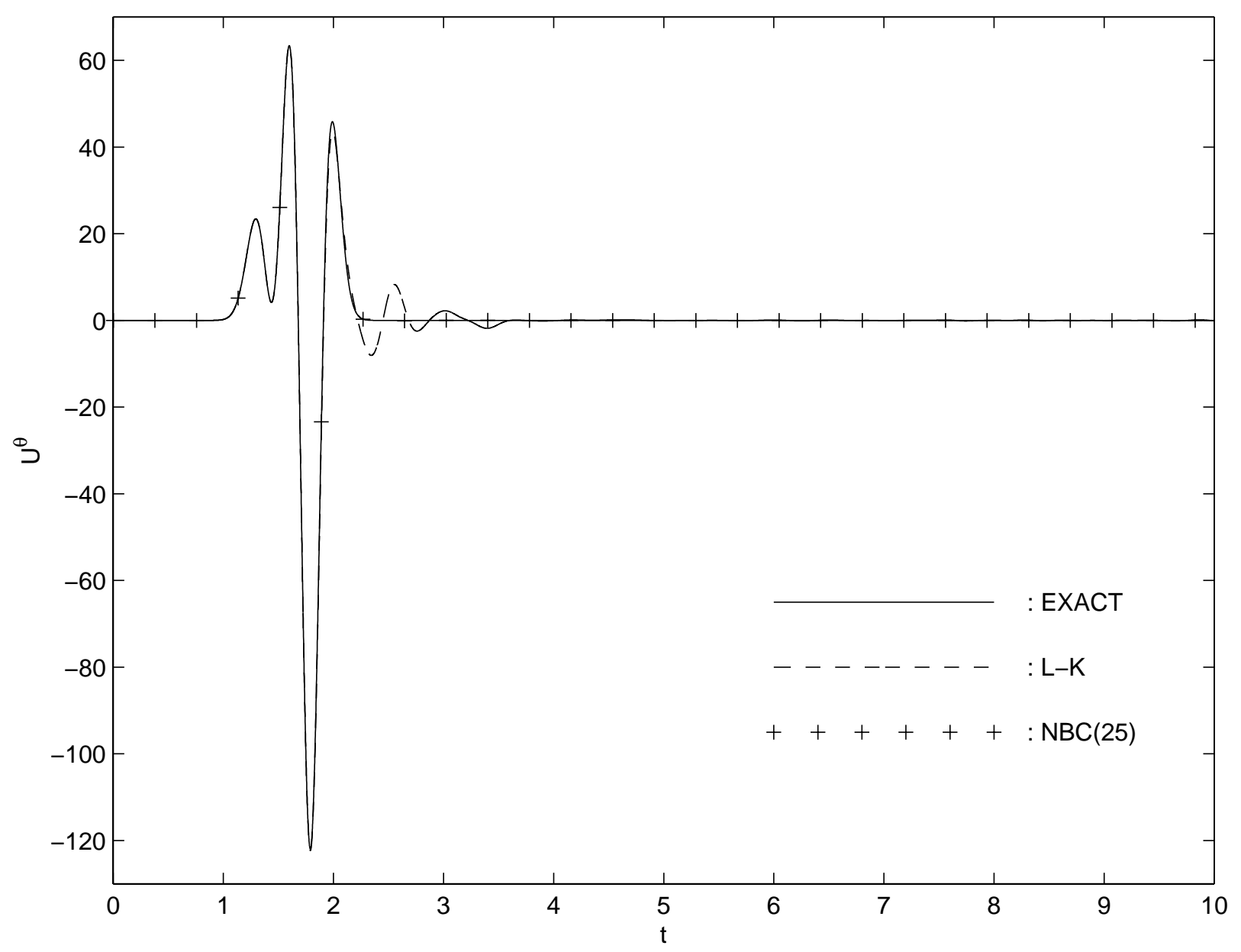

Figure 5: 


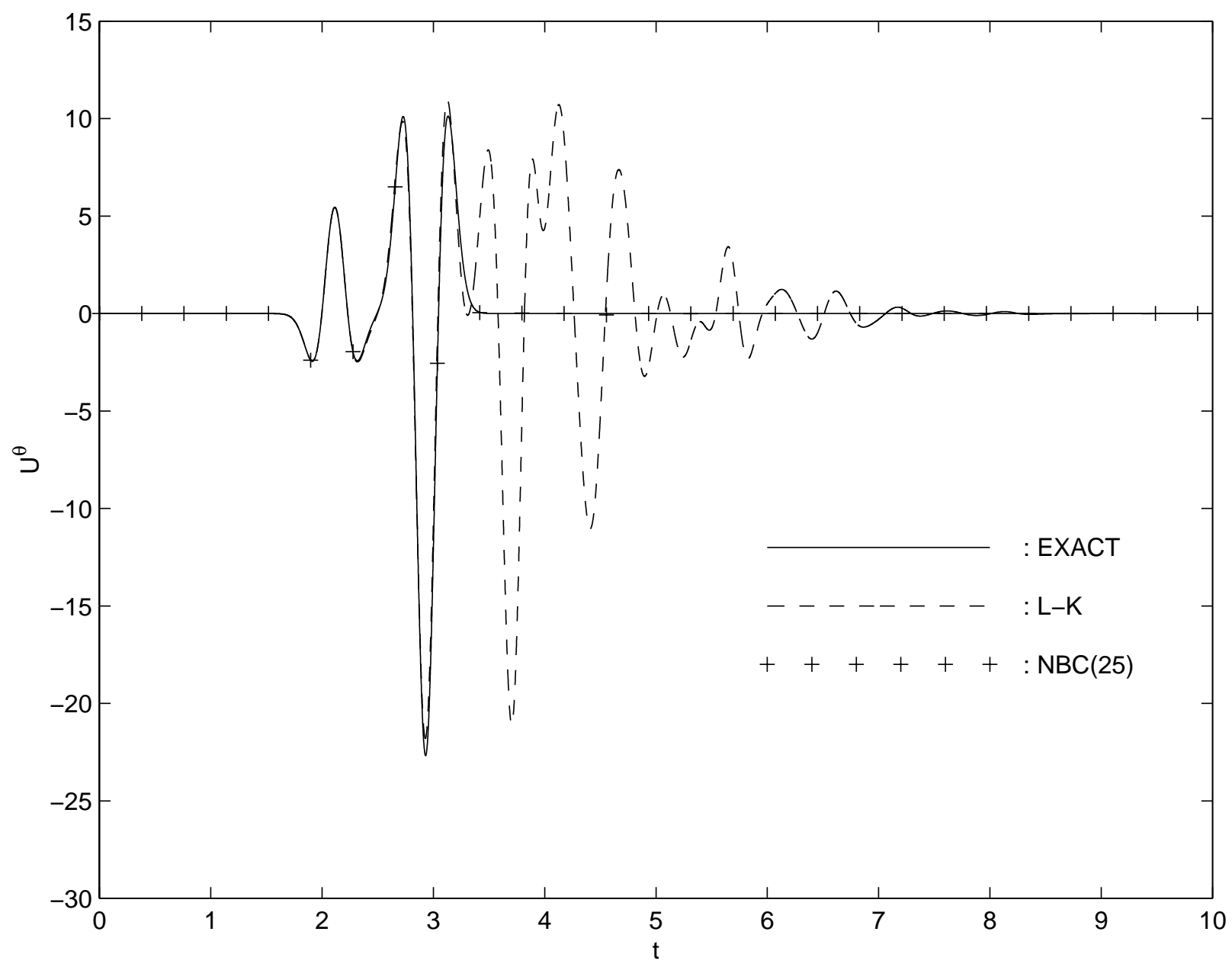

Figure 6: 


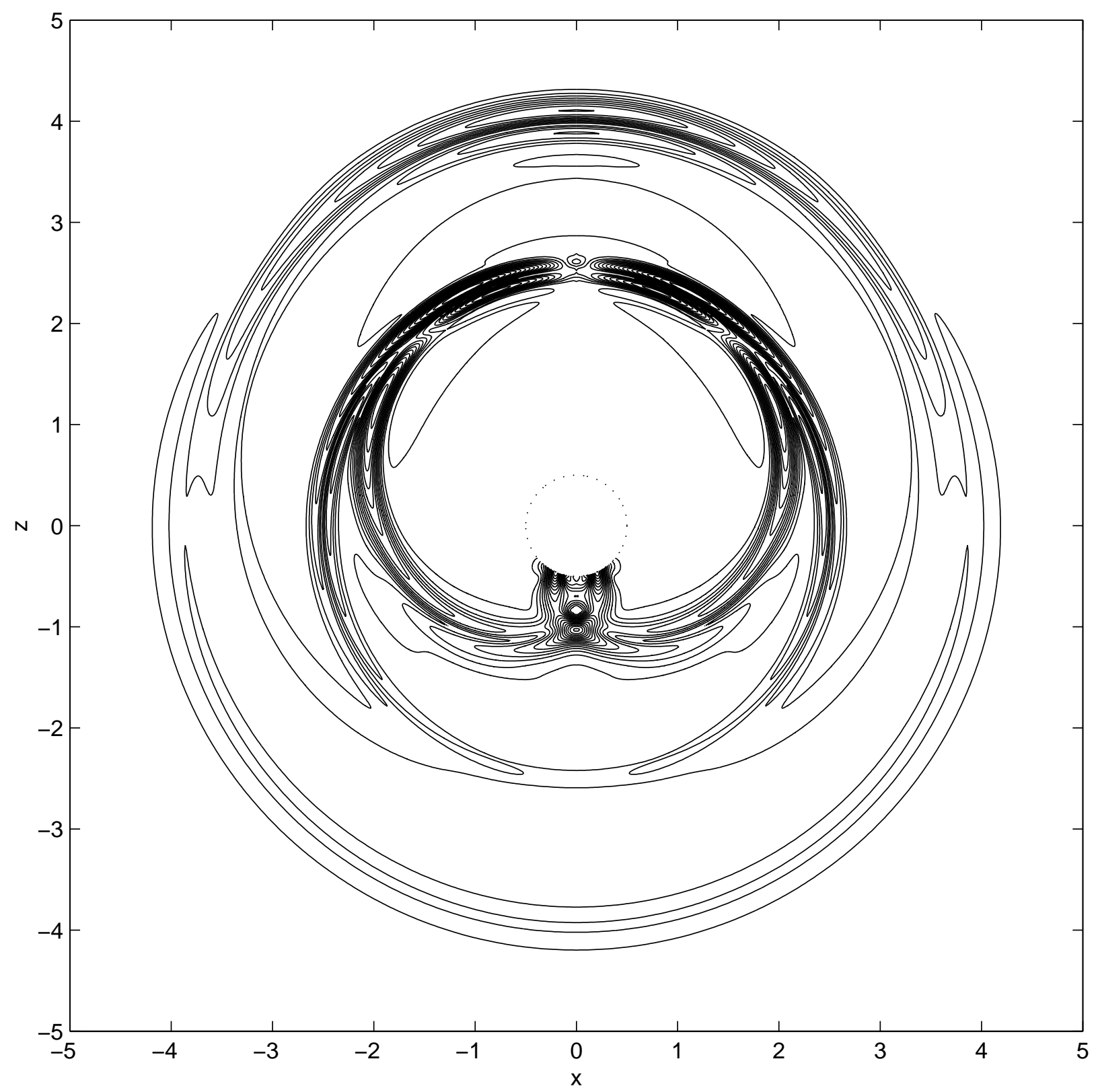

Figure 7: 


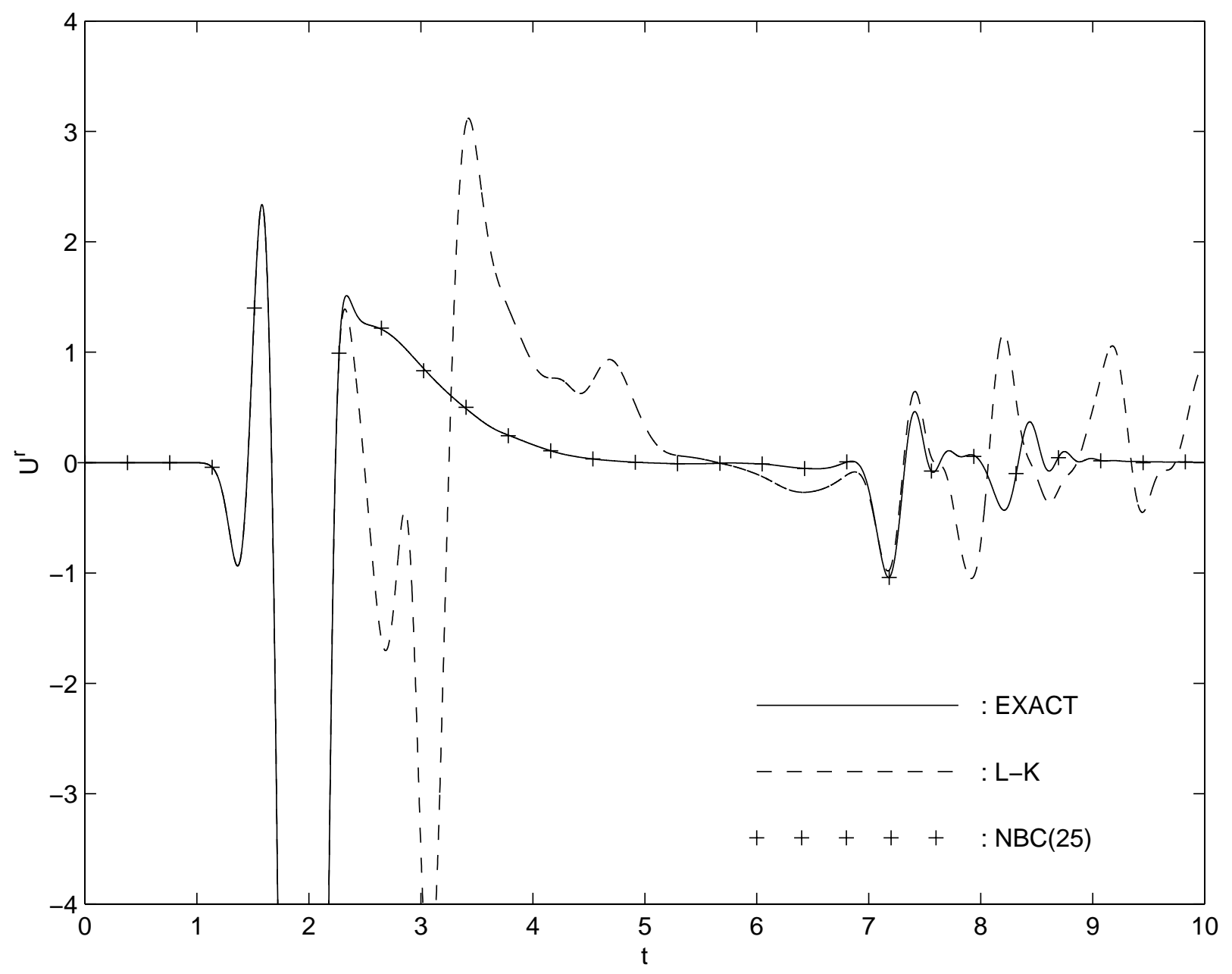

Figure 8: 


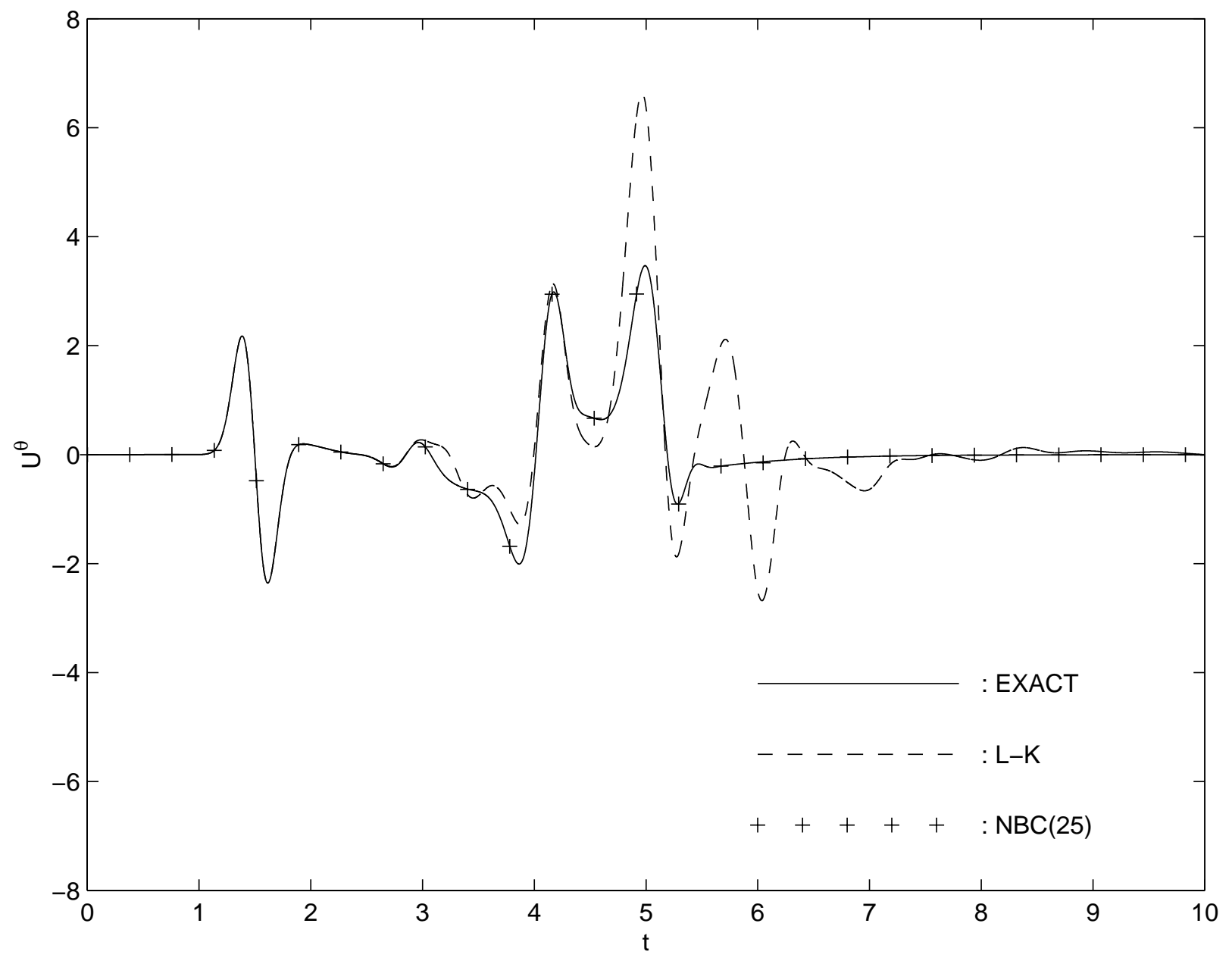

Figure 9: 


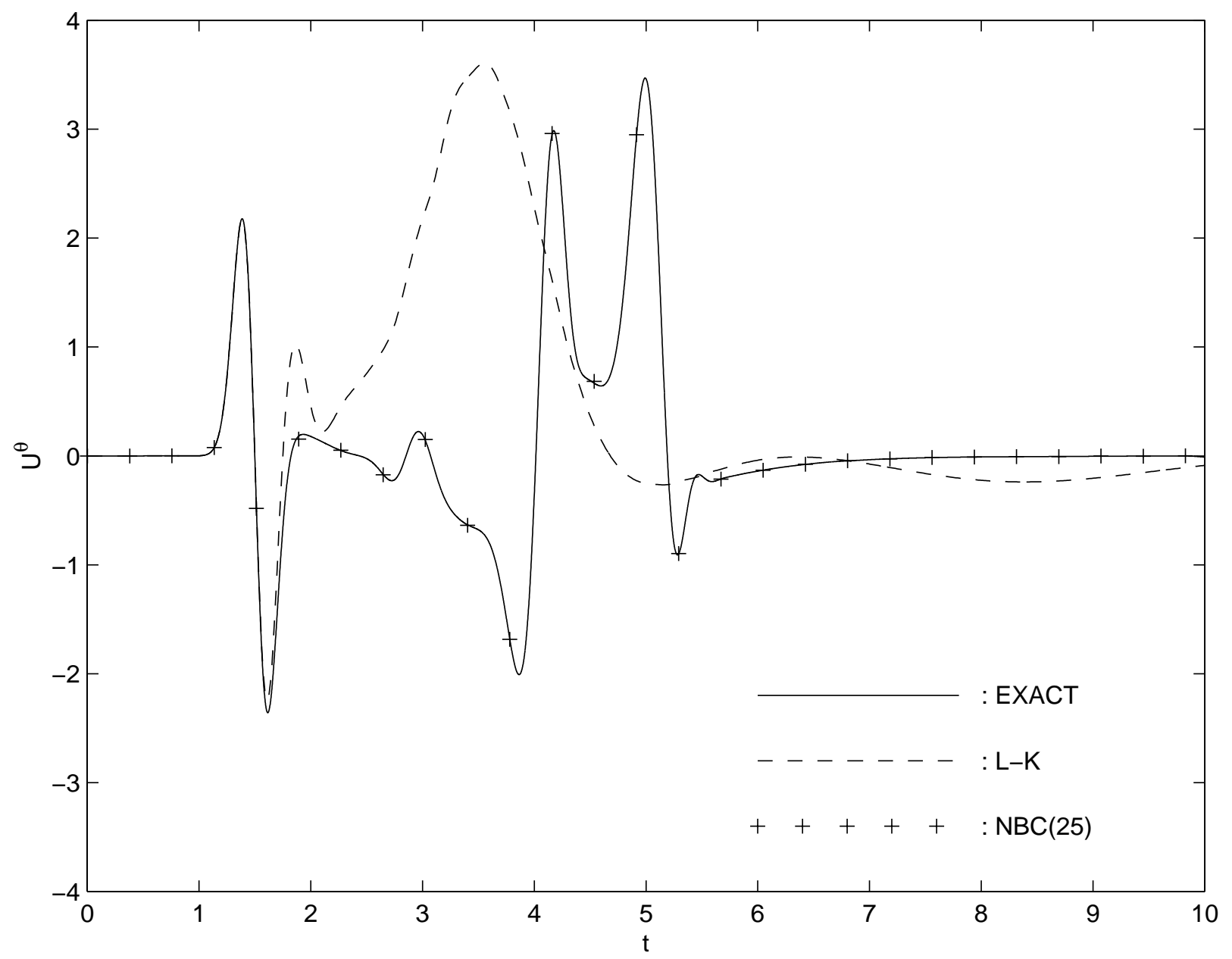

Figure 10: 